\title{
DISTRIBUTIVE LAWS VIA ADMISSIBILITY
}

\author{
CHARLES WALKER
}

\begin{abstract}
This paper concerns the problem of lifting a $\mathrm{KZ}$ doctrine $P$ to the 2 -category of pseudo $T$-algebras for some pseudomonad $T$. Here we show that this problem is equivalent to giving a pseudo-distributive law (meaning that the lifted pseudomonad is automatically KZ), and that such distributive laws may be simply described algebraically and are essentially unique (as known to be the case in the (co)KZ over KZ setting).

Moreover, we give a simple description of these distributive laws using Bunge and Funk's notion of admissible morphisms for a KZ doctrine (the principal goal of this paper). We then go on to show that the 2-category of $\mathrm{KZ}$ doctrines on a 2-category is biequivalent to a poset.

We will also discuss here the problem of lifting a locally fully faithful KZ doctrine, which we noted earlier enjoys most of the axioms of a Yoneda structure, and show that a bijection between oplax and lax structures is exhibited on the lifted "Yoneda structure" similar to Kelly's doctrinal adjunction. We also briefly discuss how this bijection may be viewed as a coherence result for oplax functors out of the bicategories of spans and polynomials, but leave the details for a future paper.
\end{abstract}

\section{Contents}

1. Introduction 2

2. Background 3

2.1. Pseudomonads and their Algebras 4

2.2. Left Extensions and Algebraic Left Extensions 8

2.3. KZ Pseudomonads and KZ Doctrines 10

3. Pseudo-Distributive Laws over KZ Doctrines 14

3.1. Notions of Pseudo-Distributive Laws 14

3.2. The Main Theorem 17

3.3. Distributive Laws over KZ Monads to those over KZ Doctrines 17

3.4. Lifting a KZ Doctrine to Algebras via a Distributive Law 26

4. Consequences and Examples 32

4.1. The Lifted KZ Doctrines 33

4.2. The Preorder of KZ Doctrines on a 2-Category 36

4.3. Examples 38

5. Liftings of Locally Fully Faithful KZ Monads 40

5.1. Doctrinal Partial Adjunctions 40

5.2. Doctrinal "Yoneda Structures" 43

5.3. Applications and Future Work $\quad 45$

References 46

Date: May 7, 2018.

2000 Mathematics Subject Classification. 18A35, 18C15, $18 \mathrm{D} 05$.

Key words and phrases. KZ-doctrines, lax-idempotent pseudomonads, pseudo-distributive laws.

The author acknowledges the support of an Australian Government Research Training Program Scholarship. 


\section{INTRODUCTION}

It is well known that to give a lifting of a monad to the algebras of another monad is to give a distributive law [1]. More generally, to give a lifting of a pseudomonad to the pseudoalgebras of another pseudomonad is to give a pseudo-distributive law $[22,3]$. However, in this paper we are interested in the problem of lifting a Kock-Zöberlein pseudomonad $P$ (also known as a lax idempotent pseudomonad), as introduced by Kock [16] and Zöberlein [33], to the pseudoalgebras of some pseudomonad $T$. These KZ pseudomonads are a particular type of pseudomonad for which algebra structures are adjoint to units; an important example being the free cocompletion under a class of colimits $\Phi$.

But what does it mean to give a lifting of a $\mathrm{KZ}$ doctrine to the setting of pseudoalgebras such that the lifted pseudomonad is also KZ? One objective of this paper is to show that this problem is equivalent to giving a pseudo-distributive law (meaning a lifting of this pseudomonad automatically inherits the KZ structure), and consequently that such pseudo-distributive laws have a couple of simple descriptions. One simple description being purely algebraic (a generalization and simplification of a description given in [22, Section 11]), and another being a novel description purely in terms of left Kan extensions and Bunge and Funk's admissible maps of a KZ doctrine [2]. In fact, Bunge and Funk's admissible maps are a central tool in the proof of these results. We also see that these distributive laws are essentially unique, a generalization capturing [25, Theorem 7.4] and strengthening parts of [23, Prop. 4.1].

These two descriptions of a pseudo-distributive law correspond to two different descriptions of a KZ pseudomonad. The first, which from now on we call a $\mathrm{KZ}$ pseudomonad, is a well known algebraic description similar to Kock's [16]; the second, which we call a $\mathrm{KZ}$ doctrine, is to be the description in terms of left Kan extensions due to Marmolejo and Wood [25, Definition 3.1].

Bunge and Funk showed that admissibility in the setting of a KZ pseudomonad also has both an algebraic definition and a definition in terms of left Kan extensions. Indeed, Bunge and Funk defined a morphism $f$ to be admissible in the context of a KZ doctrine $P$ when $P f$ has a right adjoint [2, Definition 1.1], and showed that this notion of admissibility also has a description in terms of left Kan extensions [2, Prop. 1.5]. We refer to this as $P$-admissibility.

The central idea here is that instead of thinking about the problem of lifting a $\mathrm{KZ}$ doctrine algebraically, we think about the problem in terms of algebraic left Kan extensions. Moreover, this notion of admissibility is crucial here as it allows us to show that certain left extensions exist and are preserved.

A well known and motivating example the reader may keep in mind is the $\mathrm{KZ}$ doctrine for the free small cocompletion on locally small categories, with its lifting to the setting of monoidal categories described by Im and Kelly [10] via the Day convolution [4].

In Section 2 we give the necessary background for this paper, and recall the basic definitions of pseudomonads, pseudo algebras and morphisms between pseudo algebras. In particular, we recall the notion of a $\mathrm{KZ}$ pseudomonad and $\mathrm{KZ}$ doctrine and some results concerning them. In addition, we recall some results concerning algebraic left extensions. These notions will be used regularly throughout the paper.

In Section 3, which is the bulk of this paper, we use Bunge and Funk's notion of admissibility to generalize some results of Marmolejo and Wood concerning pseudo-distributive laws of (co)KZ doctrines over KZ doctrines, such as the simple form of such distributive laws [22, Section 11] or essential uniqueness of them [25, Theorem 7.4]. Our first improvement here is to show that an axiom concerning the 
(co)KZ doctrine may be dropped, allowing us to generalize these results to pseudodistributive laws of any pseudomonad over a KZ doctrine. For example, this level of generality allows us to capture the case studied by Im and Kelly [10]; showing that the lifting of the small cocompletion from categories to monoidal categories is essentially unique.

In addition, we use this simplification to give a simple algebraic description of a pseudo-distributive law of a pseudomonad over a $\mathrm{KZ}$ pseudomonad, consisting only of a pseudonatural transformation and three invertible modifications subject to three coherence axioms, and prove this definition is equivalent to the usual notion of pseudo-distributive law. However, the main new result of this section is a simple description of pseudo-distributive laws over a $\mathrm{KZ}$ doctrine purely in terms of left Kan extensions and admissibility.

Furthermore, through these calculations we find that in the presence of a such a distributive law, the lifting of a $\mathrm{KZ}$ doctrine $P$ to pseudo- $T$-algebras (for a pseudomonad $T$ ) is automatically a $\mathrm{KZ}$ doctrine. The proof of these results is highly technical, relying on $T$ preserving $P$-admissible maps; however, the main result of this section is simply stated in Theorem 35 .

In Section 4 we study some properties of the lifted $\mathrm{KZ}$ doctrine $\widetilde{P}$, such as classifying the $\widetilde{P}$-cocomplete $T$-algebras as those for which the underlying object is $P$-cocomplete and the algebra map separately cocontinuous, thus justifying the usual definition of algebraic cocompleteness. We also compare our results to that of Im-Kelly [10], but seen from the KZ doctrine viewpoint.

After checking that the 2-category of $\mathrm{KZ}$ doctrines on a 2-category is biequivalent to a poset, we go on to give some examples in which we apply our results. Our first example concerns the case of the small cocompletion and monoidal categories, and our second example concerns multi-adjoints as studied by Diers [6].

In Section 5 we consider the problem of lifting a locally fully faithful KZ doctrine. These locally fully faithful $\mathrm{KZ}$ doctrines are of interest as they almost give rise to Yoneda structures [29]. In particular, it is the goal of this section to describe a bijection between oplax and lax structures on the lifted "Yoneda structure" when we have such a distributive law; that is a bijection between cells $\alpha$ exhibiting $L$ as an oplax $T$-morphism

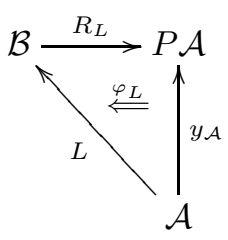

$$
\begin{aligned}
(\mathcal{B}, T \mathcal{B} \stackrel{y}{\rightarrow} \mathcal{B}) \stackrel{\left(R_{L}, \beta\right)}{\longrightarrow}\left(P \mathcal{A}, T P \mathcal{A} \stackrel{z_{x}}{\rightarrow} P \mathcal{A}\right) \\
(\mathcal{A}, T \mathcal{A} \stackrel{x}{\rightarrow} \mathcal{A})
\end{aligned}
$$

and cells $\beta$ exhibiting $R_{L}$ as a lax $T$-morphism for diagrams as on the right above, underlain by a "Yoneda structure" diagram such as that on the left above. As an instance of this result we recover Kelly's bijection between oplax structures on left adjoints and lax structures on right adjoints [13]. An interesting application of this bijection is as a coherence result for the bicategories of spans and polynomials (and in particular the oplax functors out of these bicategories). We briefly discuss the applications here, but leave this to be explored in more detail in a forthcoming paper.

\section{BACKGROUND}

It is the purpose of this section to give the background knowledge necessary for this paper. We start off by recalling the basic definitions of pseudomonads, pseudo algebras, and morphisms between pseudo algebras, as these notions will be used 
regularly throughout the paper. We then recall the notion of a left extension in a 2-category, and consider when these left extensions lift to the setting of pseudoalgebras and morphisms between them (in a sense which will be applicable in later sections). Finally, we go on to recall the notion of a KZ pseudomonad, a special type of pseudomonad for which the algebra structure maps are adjoint to units, and give their basic properties and some examples.

2.1. Pseudomonads and their Algebras. In order to define pseudomonads, we first need the notions of pseudonatural transformations and modifications. The notion of pseudonatural transformation is the (weak) 2-categorical version of natural transformation. There are weaker notions also of lax and oplax natural transformations, however those will not be used here. Modifications, defined below, take the place of morphisms between pseudonatural transformations.

Definition 1. A pseudonatural transformation between pseudofunctors $t: F \rightarrow$ $G: \mathscr{A} \rightarrow \mathscr{B}$ where $\mathscr{A}$ and $\mathscr{B}$ are bicategories provides for each 1-cell $f: \mathcal{A} \rightarrow \mathcal{B}$ in $\mathscr{A}, 1$-cells $t_{\mathcal{A}}$ and $t_{\mathcal{B}}$ and an invertible 2 -cell $t_{f}$ in $\mathscr{B}$ as below

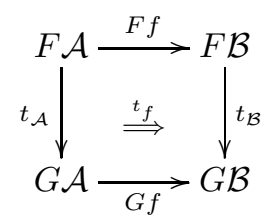

satisfying coherence conditions outlined in [14, Definition 2.2]. Given two pseudonatural transformations $t, s: F \rightarrow G: \mathscr{A} \rightarrow \mathscr{B}$ as above, a modification $\alpha: s \rightarrow t$ consists of, for every object $\mathcal{A} \in \mathscr{A}$, a 2-cell $\alpha_{\mathcal{A}}: t_{\mathcal{A}} \rightarrow s_{\mathcal{A}}$ such that for each 1-cell $f: \mathcal{A} \rightarrow \mathcal{B}$ in $\mathscr{A}$ we have the equality $\alpha_{\mathcal{B}} \cdot F f \circ t_{f}=s_{f} \circ G f \cdot \alpha_{\mathcal{A}}$.

The following defines the (weak) 2-categorical version of monad to be used throughout this paper. For brevity, we will suppress pseudofunctoriality constraints in this definition and those following.

Definition 2. A pseudomonad on a 2-category $\mathscr{C}$ consists of a pseudofunctor equipped with pseudonatural transformations as below

$$
T: \mathscr{C} \rightarrow \mathscr{C}, \quad u: 1_{\mathscr{C}} \rightarrow T, \quad m: T^{2} \rightarrow T
$$

along with three invertible modifications
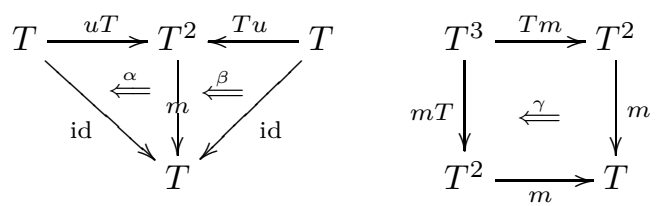

subject to the two coherence axioms
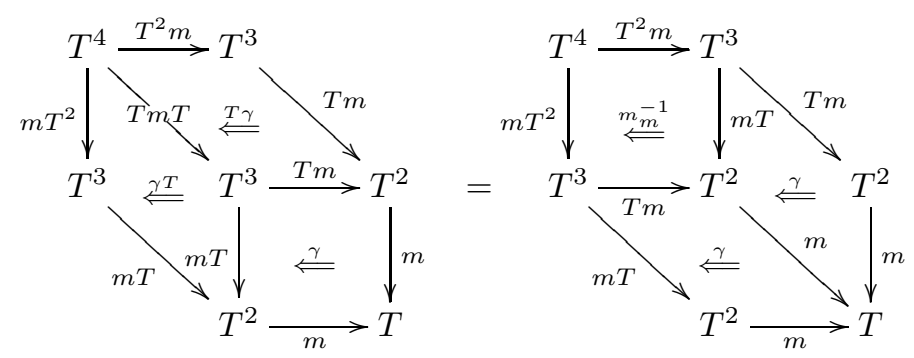
and

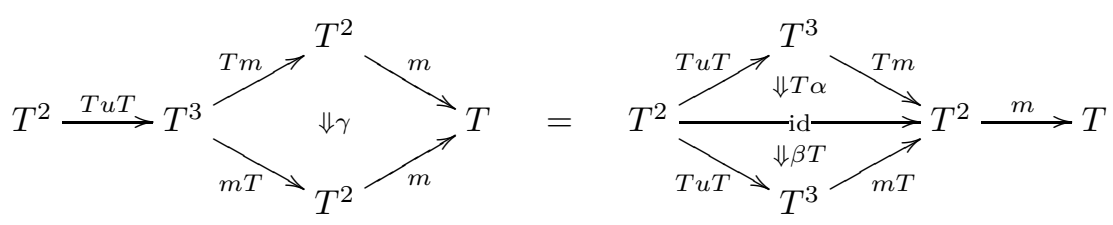

Remark 3. One should note here that there are three useful consequences of these pseudomonad axioms [21, Proposition 8.1] originally due to Kelly [11]. Of these, we will only need the consequence that

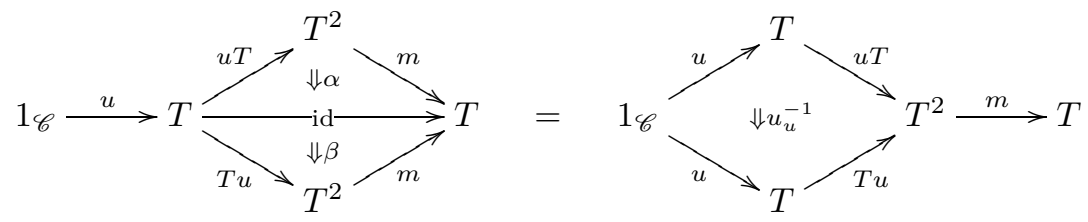

Given a pseudomonad $(T, u, m)$ on a 2 -category $\mathscr{C}$ one may consider its strict $T$ algebras and strict $T$-morphisms, or the weaker counterparts where conditions only hold up coherent 2-cells. These weaker notions are what will be used throughout this paper, though usually with the coherent 2-cells in question being invertible. For convenience, we will leave the modifications $\alpha, \beta$ and $\gamma$ in the above definition as unnamed isomorphisms throughout the rest of the paper.

Definition 4. Given a pseudomonad $(T, u, m)$ on a 2-category $\mathscr{C}$, a lax $T$-algebra consists of an object $\mathcal{A} \in \mathscr{C}$, a 1-cell $x: T \mathcal{A} \rightarrow \mathcal{A}$ and 2-cells
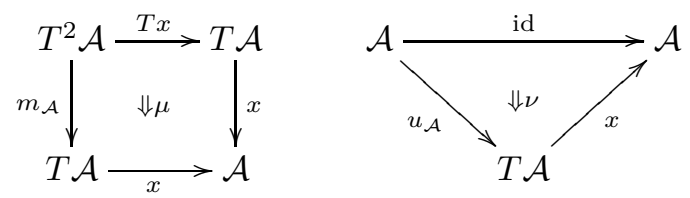

such that both
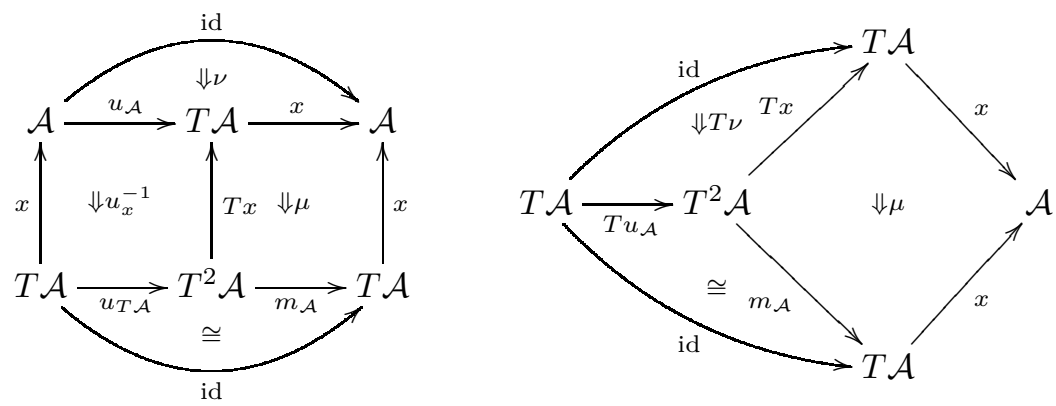

paste to the identity 2 -cell at $x$, known as the left and right unit axioms respectively. Moreover, the associativity axiom asks that we have the equality
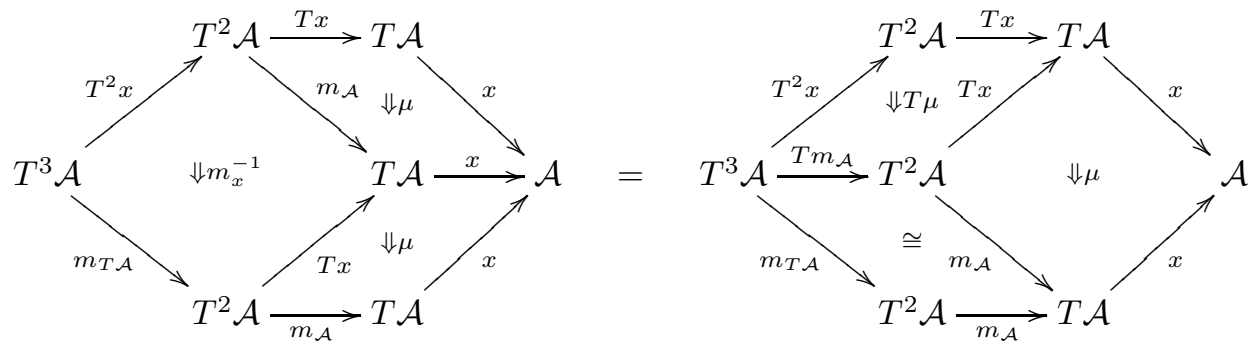
If the above 2-cells $\nu$ and $\mu$ are isomorphisms, we call this a pseudo T-algebra. If $\nu$ and $\mu$ are identity 2 -cells, we call this a strict $T$-algebra.

These $T$-algebras may be regarded as the objects of a category, with morphisms of (pseudo) $T$-algebras defined as follows.

Definition 5. Given a pseudomonad $(T, u, m)$ on a 2-category $\mathscr{C}$, an oplax $T$ morphism of pseudo $T$-algebras

$$
(L, \alpha):(\mathcal{A}, T \mathcal{A} \stackrel{x}{\rightarrow} \mathcal{A}) \rightarrow(\mathcal{B}, T \mathcal{B} \stackrel{y}{\rightarrow} \mathcal{B})
$$

consists of a 1-cell $L: \mathcal{A} \rightarrow \mathcal{B}$ and a 2-cell

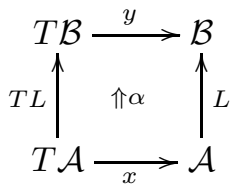

such that (leaving the pseudo $T$-algebra coherence cells as unnamed isomorphisms)

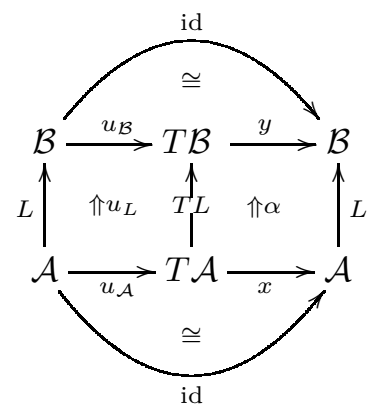

is the identity 2 -cell on $L$, and for which

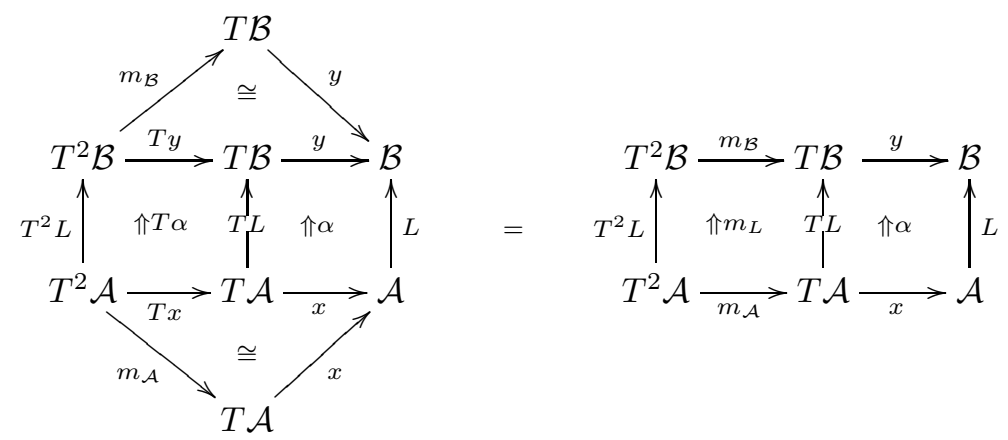

If the 2-cell $\alpha$ goes in the opposite direction, this is the definition of a lax $T$ morphism, and if $\alpha$ is invertible this is then the definition of a pseudo T-morphism.

The usual definition of $T$-transformation between oplax or lax $T$-morphisms is not general enough for our purposes as we will be considering situations in which we have both oplax and lax $T$-morphisms, and so we define $T$-transformations as based on the double category viewpoint [8]. Such transformations are sometimes referred to as generalized $T$-transformations. 
Definition 6. Suppose we are given a square of morphisms of pseudo $T$-algebras

$$
\begin{array}{ccc}
(\mathcal{B}, y) & \stackrel{(R, \beta)}{\longrightarrow}(\mathcal{C}, z) \\
(N, \varphi) \uparrow & \stackrel{\zeta}{\longleftarrow} & \uparrow(I, \xi) \\
(\mathcal{D}, w) & \stackrel{(M, \varepsilon)}{\rightleftarrows}(\mathcal{A}, x)
\end{array}
$$

where the vertical maps are oplax $T$-morphisms and the horizontal maps are lax $T$ morphisms. A T-transformation $\zeta$ as in the above square is a 2-cell $\zeta: I \cdot M \rightarrow R \cdot N$ for which we have the equality of the two sides of the cube
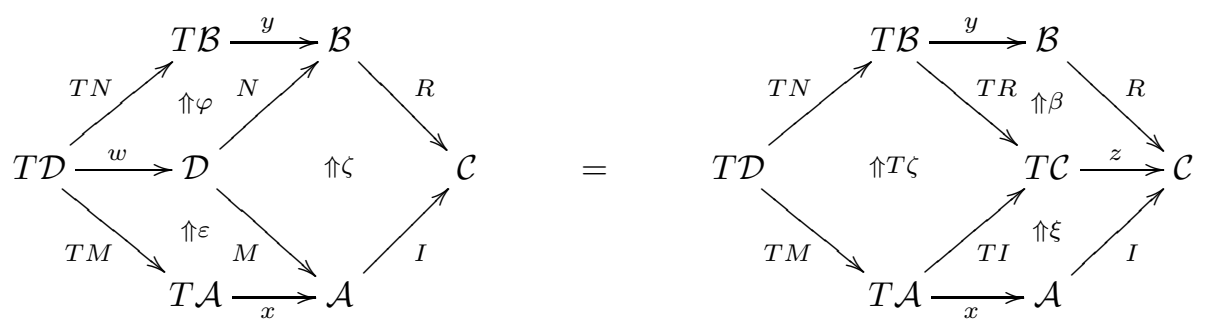

We will call the 2-category of pseudo $T$-algebras, pseudo $T$-morphisms, and $T$ transformations ps- $T$-alg (we may consider squares where both horizontal maps are identities or both vertical maps are identities to recover the usual notions of transformation between lax/oplax/pseudo $T$-morphisms).

Remark 7. Note that in this language it makes sense to talk about the unit and counit of an adjunction where the left adjoint is oplax and the right adjoint lax. Indeed the oplax-lax bijective correspondence in Kelly's doctrinal adjunction [13] is unique such the counit $\varepsilon$ (and unit $\eta$ ) of the adjunction is a $T$-transformation ${ }^{1}$. Note also that in this setting of a doctrinal adjunction $L \dashv R$ (with an oplax structure $\alpha$ on $L$ corresponding a lax structure $\beta$ on $R$ via the mates correspondence) it makes sense to view the unit and counit as $T$-transformations as we have squares

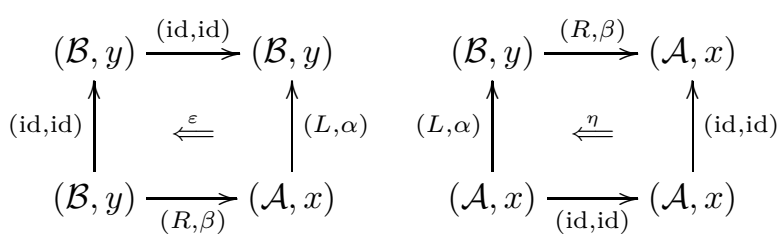

As a convention, will will usually omit these identity $T$-morphisms. The reader may just remember that it makes sense to consider $T$-transformations from a lax followed by an oplax $T$-morphism, into an oplax followed by a lax $T$-morphism, and that any such transformation may be uniquely expressed as a square in the form of the above definition by inserting the appropriate identity $T$-morphisms; which is what we have done in the case of the unit and counit above.

Example 8. One may define the category of Cat (the category of locally small categories) enriched graphs, denoted CatGrph, with objects given as families of hom-categories

$$
(\mathscr{C}(X, Y): X, Y \in \mathrm{ob} \mathscr{C})
$$

and morphisms consisting of locally defined functors

$$
\left(F_{X, Y}: \mathscr{C}(X, Y) \rightarrow \mathscr{D}(F X, F Y): X, Y \in \mathscr{C}\right)
$$

\footnotetext{
${ }^{1}$ This is shown in more generality in Proposition 72 .
} 
which have not been endowed with the structure of a bicategory or a lax/oplax functor respectively [18]. This gives rise to, via a suitable 2-monad $T$ on CatGrph, the 2-category of bicategories, oplax functors and icons [19]. We may of course replace oplax here with "lax" or "pseudo". Note that inside this 2-category lives the one object bicategories (isomorphic to monoidal categories), giving the 2-category of monoidal categories, lax/oplax/strong monoidal functors and monoidal transformations (which may also be constructed directly via a suitable 2-monad [19]).

2.2. Left Extensions and Algebraic Left Extensions. In this section we will consider how pseudomonads interact with left extensions. In particular, we start off by recalling the notion of a left extension in a 2-category, and go on to give conditions under which such a left extension lifts to a suitable notion of left extension in the setting of pseudo $T$-algebras, $T$-morphisms and $T$-transformations. The results of this section are mostly due to Koudenburg, shown in a more general double category setting [17].

Definition 9. Suppose we are given a 2-cell $\eta: I \rightarrow R \cdot L$ as in the left diagram
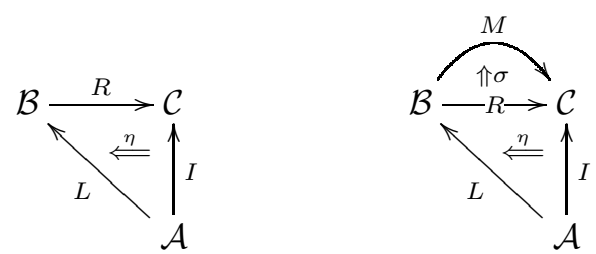

in a 2-category $\mathscr{C}$. We say that $R$ is exhibited as a left extension of $I$ along $L$ by the 2-cell $\eta$ when pasting 2-cells $\sigma: R \rightarrow M$ with the 2-cell $\eta: I \rightarrow R \cdot L$ as in the right diagram defines a bijection between 2-cells $R \rightarrow M$ and 2-cells $I \rightarrow M \cdot L$. Moreover, we say such a left extension $(R, \eta)$ is respected (also called preserved) by a 1-cell $E: \mathcal{C} \rightarrow \mathcal{D}$ when the whiskering of $\eta$ by $E$, as given by the pasting diagram below

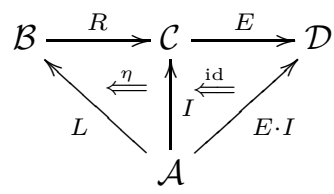

exhibits $E \cdot R$ as a left extension of $E \cdot I$ along $L$.

We now give a suitable description of when a lax $T$-morphism may be regarded as a left extension in the setting of pseudo $T$-algebras.

Definition 10. Suppose we are given an oplax $T$-morphism $(L, \alpha)$ and lax $T$ morphisms $(R, \beta)$ and $(I, \sigma)$ between pseudo $T$-algebras equipped with a $T$-transformation $\eta: I \rightarrow R \cdot L$ as in the diagram

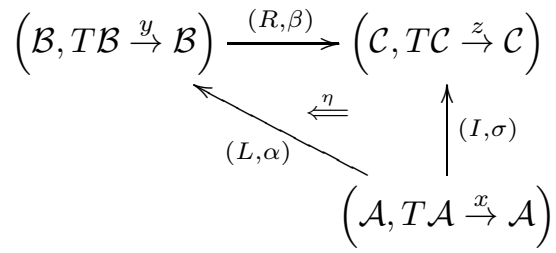


We call such a diagram a $T$-left extension if for any given pseudo $T$-algebra $(\mathcal{D}, w)$, lax $T$-morphism $(M, \varepsilon)$ and oplax $T$-morphism $(N, \varphi)$ as below

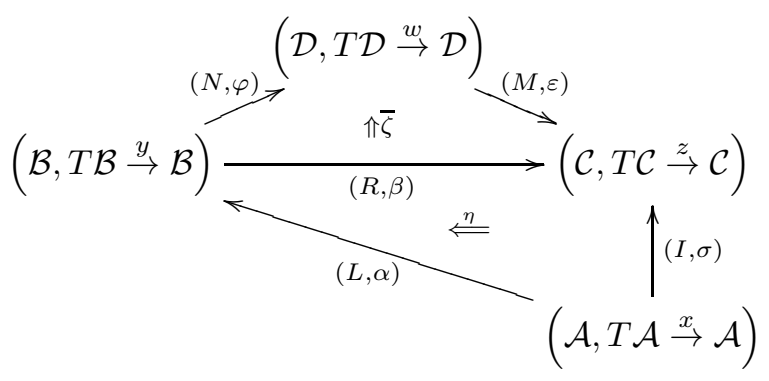

pasting $T$-transformations of the form $\bar{\zeta}$ above with the $T$-transformation $\eta$ defines the bijection of $T$-transformations:

$$
\begin{aligned}
& (\mathcal{D}, w) \stackrel{(M, \varepsilon)}{\longrightarrow}(\mathcal{C}, z) \quad(\mathcal{D}, w) \stackrel{(M, \varepsilon)}{\longrightarrow}(\mathcal{C}, z)
\end{aligned}
$$

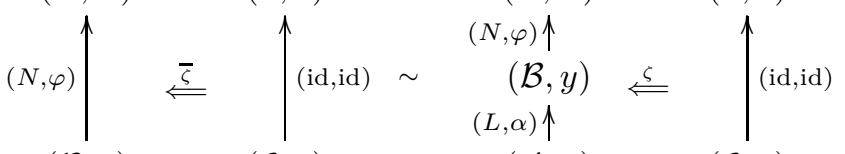

$$
\begin{aligned}
& (\mathcal{B}, y) \underset{(R, \beta)}{\longrightarrow}(\mathcal{C}, z) \quad(\mathcal{A}, x) \underset{(I, \sigma)}{\longrightarrow}(\mathcal{C}, z)
\end{aligned}
$$

Remark 11. Note that if $\bar{\zeta}$ and $\eta$ are both $T$-transformations then so is the composite $\bar{\zeta} L \cdot \eta$; this is a simple calculation which we omit.

In order to lift left extensions to $T$-left extensions as above we will require the following algebraic cocompleteness property.

Definition 12. Given a pseudomonad $(T, u, m)$ on a 2-category $\mathscr{C}$, we say a left extension $(H, \varphi)$ in $\mathscr{C}$ as on the left below is $T$-preserved by a 1 -cell $z: T \mathcal{C} \rightarrow \mathcal{D}$ when
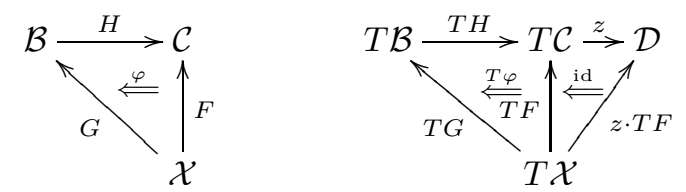

the pasting diagram on the right exhibits $(z \cdot T H, z \cdot T \varphi)$ as a left extension.

Remark 13. Given a pseudo $T$-algebra $(\mathcal{C}, T \mathcal{C} \stackrel{z}{\rightarrow} \mathcal{C})$ if we ask that the underlying object $\mathcal{C}$ is cocomplete in the sense that all left extensions (along a chosen class of maps) into $\mathcal{C}$ exist, and moreover that the algebra structure map $z T$-preserves these left extensions, then this is (essentially) the notion of algebraic cocompleteness as given by Weber [32, Definition 2.3.1] (except that we are not using pointwise left extensions here). In the setting monoidal categories, this condition of $z$ (when $z$ is an algebra structure map) $T$-preserving the left extensions is the analogue of asking the tensor product be separately cocontinuous; see [32, Prop. 2.3.2].

We now recall a result for algebraic left extensions mostly due to Koudenburg [17] (though we avoid working in a double categorical setting). We will include some details of the proof as we will need them later.

Proposition 14. Suppose we are given a diagram

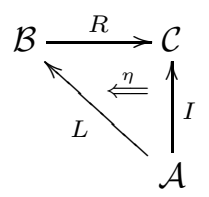


which exhibits $R$ as a left extension in a 2-category $\mathscr{C}$ equipped with a pseudomonad $(T, u, m)$. Suppose further that

$$
(\mathcal{A}, T \mathcal{A} \stackrel{x}{\longrightarrow} \mathcal{A}), \quad(\mathcal{B}, T \mathcal{B} \stackrel{y}{\longrightarrow} \mathcal{B}), \quad(\mathcal{C}, T \mathcal{C} \stackrel{z}{\longrightarrow} \mathcal{C})
$$

are pseudo T-algebras. Suppose even further that the left extension $(R, \eta)$ is $T$ preserved by $z$, and the resulting left extension $(z \cdot T R, z \cdot T \eta)$ is itself $T$-preserved by $z$. Then given a lax T-morphism structure $\sigma$ on $I$ and an oplax T-morphism structure $\alpha$ on $L$, there exists a unique lax $T$-morphism structure $\beta$ on $R$ for which $\eta$ is a T-transformation. Moreover, this left extension is then lifted to the T-left extension

$$
\begin{aligned}
& (\mathcal{B}, T \mathcal{B} \stackrel{y}{\rightarrow} \mathcal{B}) \underset{(L, \alpha)}{\stackrel{(R, \beta)}{\longrightarrow}}(\mathcal{C}, T \mathcal{C} \stackrel{z}{\rightarrow} \mathcal{C}) \\
& (\mathcal{A}, T \mathcal{A} \stackrel{x}{\rightarrow} \mathcal{A})
\end{aligned}
$$

Proof. Given our structure cells $\sigma$ and $\alpha$ as below
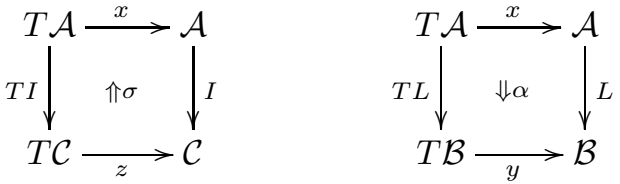

our lax constraint cell for $R$ is given as the unique $\beta$ such that $\eta$ is a $T$-transformation, that is the unique 2-cell such that
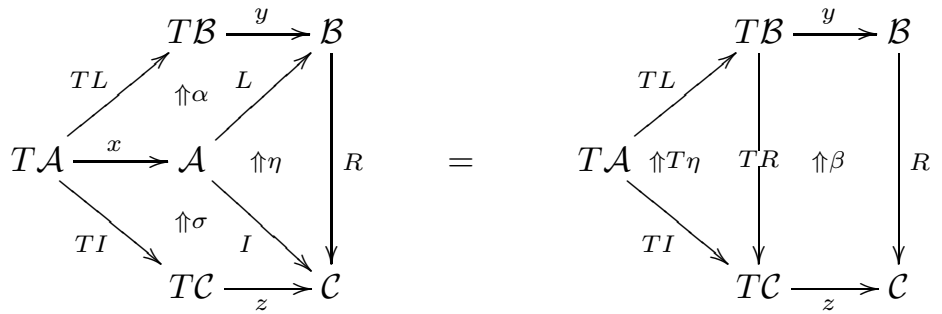

as $z \cdot T \eta$ exhibits $z \cdot T R$ as a left extension. From here, the proof of the coherence axioms for $\beta$ being a lax $T$-morphism structure on $R$ is the same as in [32, Theorem 2.4.4 $]^{2}$. Checking that the lax $T$-morphism $(R, \beta)$ is then a $T$-left extension is a straightforward exercise, of which we omit the details.

2.3. KZ Pseudomonads and KZ Doctrines. A KZ pseudomonad is a special type of pseudomonad for which the algebra structure maps are adjoint to units; with typical examples including the cocompletion of a category under some class of colimits $\Phi$. For this paper, we will use two different (but equivalent) characterizations of KZ pseudomonads. The first characterization we will use is a well known algebraic description of a $\mathrm{KZ}$ pseudomonad, described via conditions on a "KZ structure cell" (similar to [16]), the second characterization is in terms of left extensions, and will be referred to as a $\mathrm{KZ}$ doctrine.

Remark 15. Note that there are other (still equivalent) characterizations which may be referred to as KZ pseudomonads or KZ doctrines. For example the characterization through adjoint strings [21], or the characterization as lax idempotent pseudomonads [15].

\footnotetext{
${ }^{2}$ The assumptions of [32, Theorem 2.4.4] concerning comma objects are not required for the proof of the coherence axioms.
} 
Definition 16. A $K Z$ pseudomonad $(P, y, \mu)$ on a 2-category $\mathscr{C}$ consists of a pseudomonad $(P, y, \mu)$ on $\mathscr{C}$ along with a modification $\theta: P y \rightarrow y P$ for which

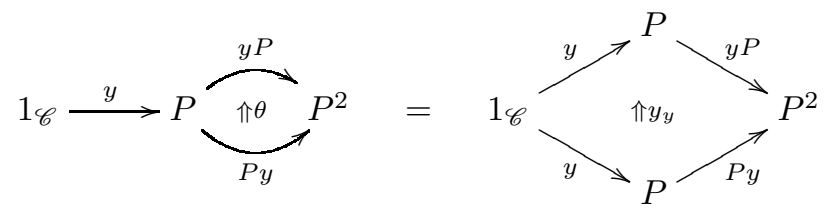

and

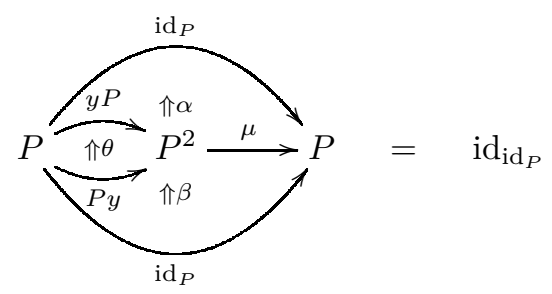

Remark 17. It is shown in [21, Prop. 3.1, Lemma 3.2] that given the adjoint string characterization we recover the definition given above, and conversely given the above definition it is not hard to recover the adjoint string definition, especially since it suffices to give only one adjunction [21, Theorem 11.1].

The above is an algebraic description of a KZ pseudomonad; however there is another description in terms of left Kan extensions given by Marmolejo and Wood [25] which we refer to as a $\mathrm{KZ}$ doctrine.

Definition 18. [25, Definition 3.1] A $K Z$ doctrine $(P, y)$ on a 2-category $\mathscr{C}$ consists of

(i) An assignation on objects $P: \mathrm{ob} \mathscr{C} \rightarrow \mathrm{ob} \mathscr{C}$;

(ii) For every object $\mathcal{A} \in \mathscr{C}$, a 1 -cell $y_{\mathcal{A}}: \mathcal{A} \rightarrow P \mathcal{A}$;

(iii) For every pair of objects $\mathcal{A}$ and $\mathcal{B}$ and 1-cell $F: \mathcal{A} \rightarrow P \mathcal{B}$, a left extension

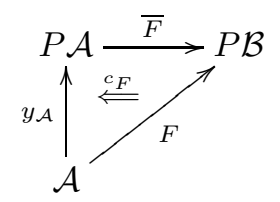

of $F$ along $y_{\mathcal{A}}$ exhibited by an isomorphism $c_{F}$ as above.

Moreover, we require that:

(a) For every object $\mathcal{A} \in \mathscr{C}$, the left extension of $y_{\mathcal{A}}$ as in 2.4 is given by

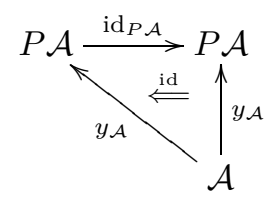

Note that this means $c_{y_{\mathcal{A}}}$ is equal to the identity 2-cell on $y_{\mathcal{A}}$.

(b) For any 1-cell $G: \mathcal{B} \rightarrow P \mathcal{C}$, the corresponding left extension $\bar{G}: P \mathcal{B} \rightarrow P \mathcal{C}$ preserves the left extension $\bar{F}$ in 2.4 .

Remark 19. These two descriptions are equivalent in the sense that each gives rise to the other $[25,21]$. In Section 4 we will express this relationship as a biequivalence between the 2-category of $\mathrm{KZ}$ pseudomonads and the preorder of $\mathrm{KZ}$ doctrines.

The following definitions in terms of left extensions are equivalent to the preceding notions of pseudo $P$-algebra and $P$-homomorphism, in the sense that we have 
an equivalence between the two resulting 2-categories of pseudo $P$-algebras arising from the two different definitions [25, Theorems 5.1,5.2].

Definition 20 ([25]). Given a $\mathrm{KZ}$ doctrine $(P, y)$ on a 2-category $\mathscr{C}$, we say an object $\mathcal{X} \in \mathscr{C}$ is $P$-cocomplete if for every $G: \mathcal{B} \rightarrow \mathcal{X}$
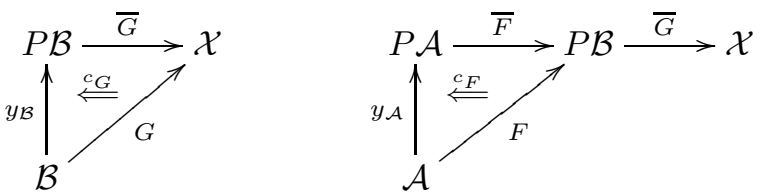

there exists a left extension $\bar{G}$ as on the left exhibited by an isomorphism $c_{G}$, and moreover this left extension respects the left extensions $\bar{F}$ as in the diagram on the right. We say a 1-cell $E: \mathcal{X} \rightarrow \mathcal{Y}$ between $P$-cocomplete objects $\mathcal{X}$ and $\mathcal{Y}$ is a $P$-homomorphism (also called $P$-cocontinuous) when it preserves all left extensions along $y_{\mathcal{B}}$ into $\mathcal{X}$ for every object $\mathcal{B}$.

Remark 21. It is clear that $P \mathcal{A}$ is $P$-cocomplete for every $\mathcal{A} \in \mathscr{C}$.

We now recall the notion of $P$-admissibility in the setting of a $\mathrm{KZ}$ doctrine $P$. This notion of admissibility is useful for showing that certain left extensions exist, and moreover are preserved. Note that this notion will be used regularly throughout the paper.

Definition 22. Given a $\mathrm{KZ}$ doctrine $(P, y)$ on a 2 -category $\mathscr{C}$, we say a 1-cell $L: \mathcal{A} \rightarrow \mathcal{B}$ is $P$-admissible if any of the following equivalent conditions are met:

(1) In the left diagram below
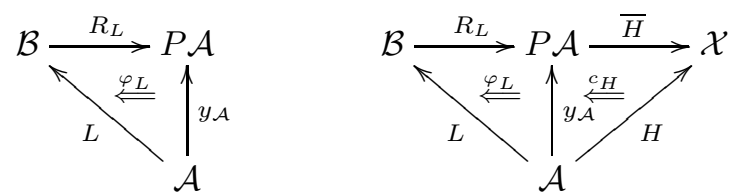

there exists a left extension $\left(R_{L}, \varphi_{L}\right)$ of $y_{\mathcal{A}}$ along $L$, and moreover the left extension is preserved by any $\bar{H}$ as in the right diagram where $\mathcal{X}$ is $P$-cocomplete;

(2) Every $P$-cocomplete object $\mathcal{X} \in \mathscr{C}$ admits, and $P$-homomorphism preserves, left extensions along $L$. This says that for any given 1-cell $K: \mathcal{A} \rightarrow$ $\mathcal{X}$, where $\mathcal{X}$ is $P$-cocomplete, there exists a 1 -cell $J$ and 2 -cell $\delta$ as in the left diagram below
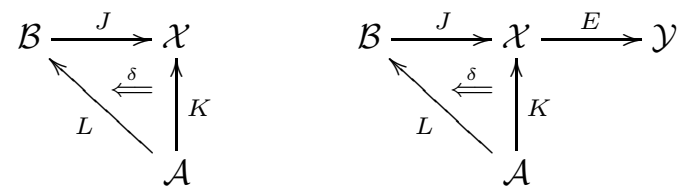

exhibiting $J$ as a left extension, and moreover this left extension is preserved by any $P$-homomorphism $E: \mathcal{X} \rightarrow \mathcal{Y}$ for $P$-cocomplete $\mathcal{Y}$ as in the right diagram;

(3) $P L:=\operatorname{lan}_{L}$ given as the left extension

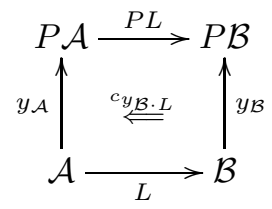

has a right adjoint. 
Remark 23. For a proof that the descriptions (1), (2) and (3) above are equivalent, we refer the reader to [2] or [29].

It is well known that pointwise left extensions along fully faithful maps are exhibited by invertible 2-cells; in the following definition we give an analogue of this fact for $\mathrm{KZ}$ doctrines.

Definition 24. Given a $\mathrm{KZ}$ doctrine $(P, y)$ on a 2 -category $\mathscr{C}$, we say a 1 -cell $L: \mathcal{A} \rightarrow \mathcal{B}$ is $P$-fully faithful if $P L$ is fully faithful.

Remark 25. The importance of the P-fully faithful maps stems from the fact that for a $P$-admissible map $L: \mathcal{A} \rightarrow \mathcal{B}$, this $L$ is $P$-fully faithful if and only if every left extension along $L$ into a $P$-cocomplete object is exhibited by an isomorphism [29, Remark 24]. Clearly each $y_{\mathcal{A}}$ is both $P$-admissible and $P$-fully faithful.

For any given $\mathrm{KZ}$ doctrine $P$ on a 2 -category $\mathscr{C}$ a natural question to ask is: what are the $P$-cocomplete objects; $P$-homomorphisms; $P$-admissible maps and $P$-fully faithful maps? Let us consider a couple of examples.

Example 26. A well known example of a $\mathrm{KZ}$ doctrine is the free small cocompletion operation on locally small categories, which sends a locally small category $\mathcal{A}$ to its category of small presheaves. In particular, when $\mathcal{A}$ is small the free small cocompletion is $P \mathcal{A}=\left[\mathcal{A}^{\mathrm{op}}\right.$, Set $]$. In this example, the $P$-cocomplete objects are those locally small categories which are small cocomplete and the $P$-homomorphisms are those functors between such categories preserving small colimits. The $P$-admissible maps are those functors $L: \mathcal{A} \rightarrow \mathcal{B}$ for which $\mathcal{B}(L-,-): \mathcal{B} \rightarrow\left[\mathcal{A}^{\text {op }}\right.$, Set $]$ factors through $P \mathcal{A}$. Of these $P$-admissible maps, the $P$-fully faithful maps are precisely the fully faithful functors.

Another example is the free large cocompletion $\mathrm{KZ}$ doctrine on locally small categories. The reader should keep in mind a theorem of Freyd showing that any locally small category which admits all large colimits is a preorder. Consequently, a locally small category is large cocomplete precisely when it is a preorder with all large joins. This KZ doctrine has some unusual properties. For example it is a cocompletion $\mathrm{KZ}$ doctrine (in the simple sense that its algebras are described as categories admitting a certain class of colimits) with unit components not always fully faithful. Moreover, every functor is admissible against the large cocompletion. We define this KZ doctrine $P$ : Cat $\rightarrow$ Cat by the assignment

$$
P: \text { ob Cat } \rightarrow \text { ob Cat }: \mathcal{A} \mapsto\left[\mathcal{A}^{\text {op }}, \mathcal{2}\right]
$$

with unit maps for each $\mathcal{A} \in$ Cat given by

$$
y_{\mathcal{A}}: \mathcal{A} \rightarrow\left[\mathcal{A}^{\mathrm{op}}, \mathcal{D}\right]: X \mapsto \mathcal{A}\langle-, X\rangle
$$

with each $\mathcal{A}\langle-, X\rangle$ is defined as

$$
\mathcal{A}\langle-, X\rangle: \mathcal{A}^{\text {op }} \rightarrow \mathcal{D}: S \mapsto \begin{cases}1, & \exists S \stackrel{f}{\longrightarrow} X \text { in } \mathcal{A} \\ 0, & \text { otherwise. }\end{cases}
$$

For any functor $F: \mathcal{A} \rightarrow \mathcal{D}$ where $\mathcal{D}$ is a preordered category with all large joins (such as $P \mathcal{B}$ for any $\mathcal{B}$ ) we may define a left extension $\bar{F}:\left[\mathcal{A}^{\text {op }}, \mathcal{Q}\right] \rightarrow \mathcal{D}$ as in the left diagram

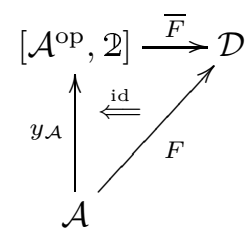

$$
\bar{F}(H)=\sup _{X \in \mathcal{A}: H X=1} F X
$$


by the assignment on the right. Hence for this $\mathrm{KZ}$ doctrine, the $P$-cocomplete objects are the large cocomplete categories, and the $P$-homomorphisms are the order and join preserving maps between such categories. Every map is $P$-admissible, and it is easily checked that a map $L: \mathcal{A} \rightarrow \mathcal{B}$ is $P$-fully faithful precisely when there exists a map $X \rightarrow Y$ in $\mathcal{A}$ if and only if there exists a map $L X \rightarrow L Y$ in $\mathcal{B}$.

Remark 27. For a set $X$ seen as a discrete category, the large cocompletion of $X$ is $(\mathscr{P} X, \supseteq)$; and dually, the large completion is $(\mathscr{P} X, \subseteq)$, where $\mathscr{P} X$ is the powerset of $X$.

\section{Pseudo-Distributive Laws over KZ Doctrines}

It was shown by Marmolejo that pseudo-distributive laws of a (co)KZ doctrine over a KZ doctrine have a particularly simple form [22, Definition 11.4]. Here we show that one can give a description which is both simpler (in that less coherence axioms are required) and more general (in that the assumption of the former pseudomonad being (co)KZ may be dropped). Hence the problem of lifting a cocompletion operation to the 2-category of pseudo algebras may be more easily understood.

Part of the motivation of our method comes from the observation that if a $\mathrm{KZ}$ doctrine lifts to a pseudomonad on the 2-category of pseudo algebras, then this pseudomonad is a $\mathrm{KZ}$ doctrine automatically ${ }^{3}$. Indeed, this fact means we may consider the problem of lifting a $\mathrm{KZ}$ pseudomonad in terms of algebraic left extensions.

In the proof we will make regular use of the admissibility perspective; in fact, the preservation of admissible maps is crucial here, and it is the main goal of this paper to describe such pseudo-distributive laws in terms of this admissibility property.

The proof of these results is quite technical, though the results are summarized in Theorem 35.

3.1. Notions of Pseudo-Distributive Laws. Beck [1] defined a distributive law of a monad $(T, u, m)$ over another monad $(P, y, \mu)$ on a category $\mathcal{C}$ to be a natural transformation $\lambda: T P \rightarrow P T$ rendering commutative the four diagrams
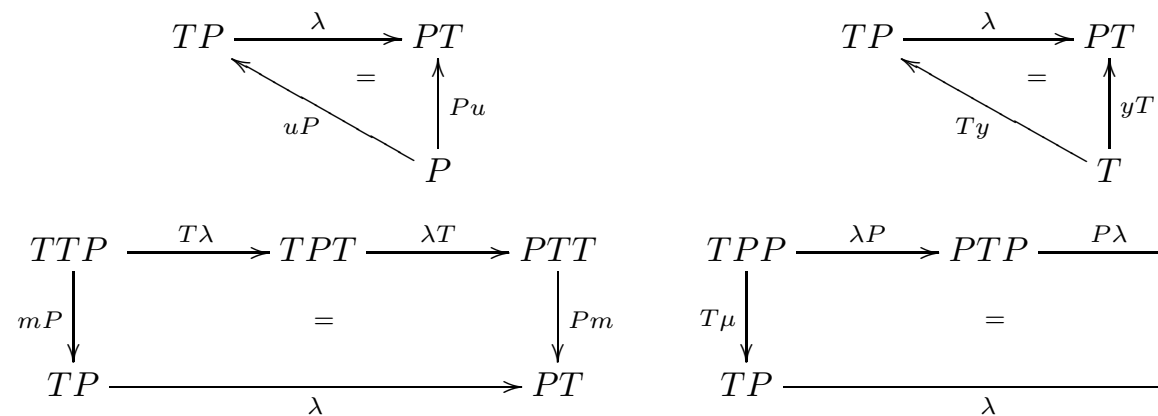

A well known example on Set is the canonical distributive law of the monad for monoids over the monad for abelian groups (whose composite is the monad for rings).

More generally, one may talk about a pseudo-distributive law of a pseudomonad over another pseudomonad on a 2-category [22, 12, 27, 3]. In this generalization the four conditions above are replaced by four pieces of data (four invertible modifications) which are then required to satisfy multiple coherence axioms, which we will omit here.

\footnotetext{
${ }^{3} \mathrm{~A}$ fact perhaps most easily seen from the adjoint string definition [21], in view of doctrinal adjunction [13].
} 
Definition 28. A pseudo-distributive law of a pseudomonad $(T, u, m)$ over a pseudomonad $(P, y, \mu)$ on a 2 -category $\mathscr{C}$ consists of a pseudonatural transformation $\lambda: T P \rightarrow P T$, along with four invertible modifications $\omega_{1}, \omega_{2}, \omega_{3}$ and $\omega_{4}$ in place of the four equalities above. These four modifications are subject to eight coherence axioms; see [24, 22].

As a convention, we choose the direction of these four modifications to be from right to left in the above four diagrams.

In this section, as in the background, we differentiate between "KZ doctrine" defined in terms of left extensions, and "KZ pseudomonad" defined algebraically.

We now define a pseudo-distributive law over such a KZ pseudomonad, though showing this data and these coherence conditions suffice will take some work.

Definition 29. Suppose we are given a 2 -category $\mathscr{C}$ equipped with a pseudomonad $(T, u, m)$ and a $\mathrm{KZ}$ pseudomonad $(P, y, \mu)$. Then a pseudo-distributive law over a KZ pseudomonad $\lambda: T P \rightarrow P T$ consists of a pseudonatural transformation $\lambda: T P \rightarrow P T$ along with three invertible modifications ${ }^{4}$
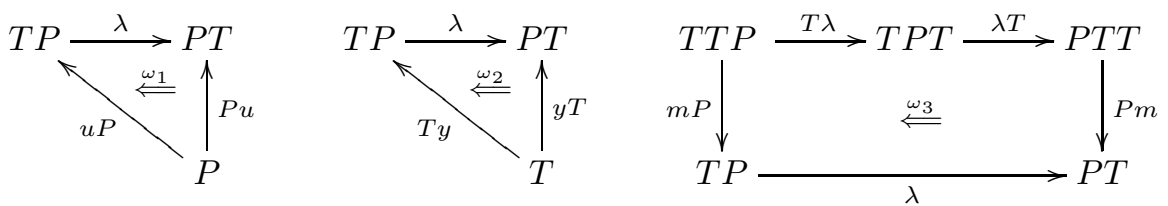

subject to the three coherence axioms:
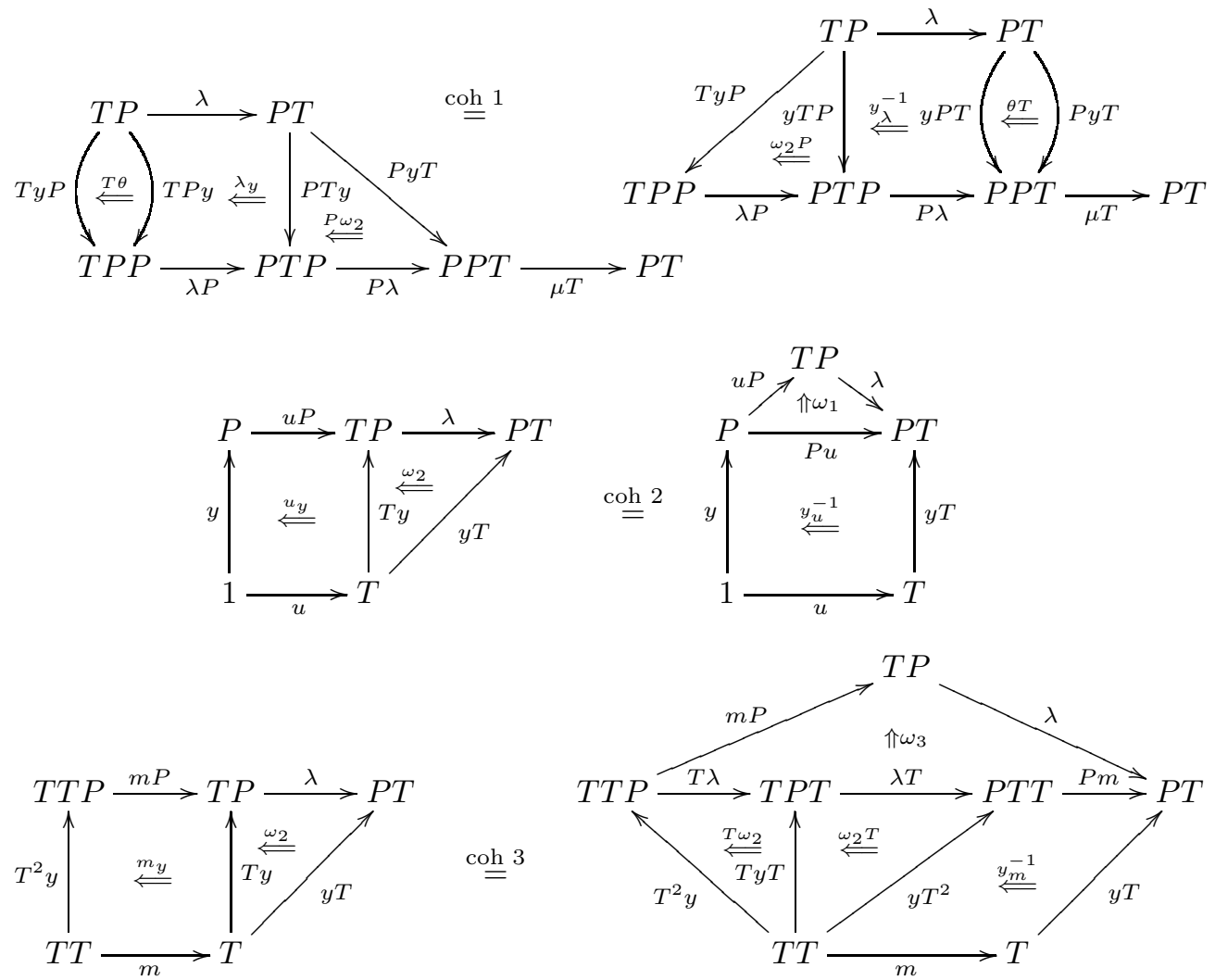

$\stackrel{\operatorname{coh}}{=} 3$

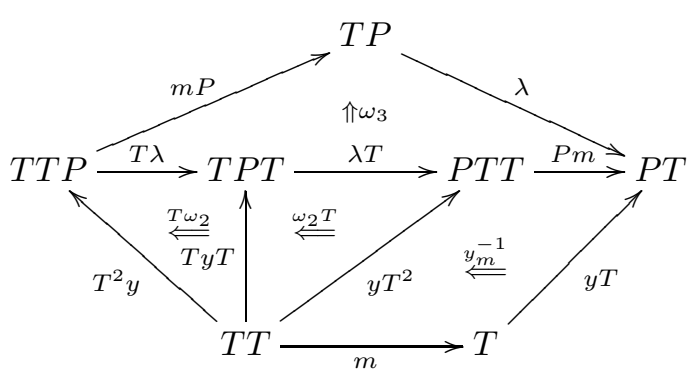

\footnotetext{
${ }^{4}$ Note the direction of the modifications are different in [22]. We use here the direction in which they will naturally arise from left extension and admissiblilty properties. Our direction agrees with that of [28, Section 4].
} 
Remark 30. (1) We will see later that $\omega_{1}$ and $\omega_{3}$ are uniquely determined by $\omega_{2}$, due to the last two axioms and left extension properties. (2) Actually, even the naturality cells of $\lambda$ may be determined given $\omega_{2}$ and the first coherence axiom. (3) With the 2-cells $\omega_{1}$ and $\omega_{3}$ and the last two coherence axioms omitted, we still have sufficient data to lift $P$ to lax $T$-algebras. (4) These last two axioms may be seen as invertibility conditions on $\omega_{1}$ and $\omega_{3}$, analogous to those in [22, Definition 11.4]. (5) During the proof, we will see that each component $\omega_{2}^{\mathcal{A}}$ necessarily exhibits each component $\lambda_{\mathcal{A}}$ as a left extension. As $\omega_{2}$ uniquely determines the rest of the data, this will show that such pseudo-distributive laws are essentially unique. (6) In fact, the first coherence axiom above is equivalent to preservation of admissible maps, in the presence of such a pseudonatural transformation $\lambda$ and invertible modification $\omega_{2}$.

We will need a notion of separately cocontinuous in the context of KZ doctrines, and so we define the following.

Definition 31. Suppose we are given a 2 -category $\mathscr{C}$ equipped with a pseudomonad $(T, u, m)$ and a $\mathrm{KZ}$ doctrine $(P, y)$. We define a 1 -cell $z: T \mathcal{X} \rightarrow \mathcal{C}$ where $\mathcal{X}$ and $\mathcal{C}$ are $P$-cocomplete objects to be:

(1) $T_{P}$-cocontinuous when every left extension along a unit component $y_{\mathcal{A}}: \mathcal{A} \rightarrow$ $P \mathcal{A}$ into $\mathcal{X}$ is $T$-preserved by $z$;

(2) $T_{P}$-adm-cocontinuous when every left extension along a $P$-admissible map $L: \mathcal{A} \rightarrow \mathcal{B}$ into $\mathcal{X}$ is $T$-preserved by $z$;

Remark 32. We will see later in Proposition 47 that these two notions are equivalent in the presence of a pseudo-distributive law of $T$ over $P$.

We are now ready to give the definition of a pseudo-distributive law over a $\mathrm{KZ}$ doctrine in terms of admissibility and left extensions.

Definition 33. Suppose we are given a 2 -category $\mathscr{C}$ equipped with a pseudomonad $(T, u, m)$ and a KZ doctrine $(P, y)$. Then a pseudo-distributive law over a $K Z$ doctrine $\lambda: T P \rightarrow P T$ consists of the following assertions:

(1) $T$ preserves $P$-admissible maps; and for every $\mathcal{A} \in \mathscr{C}$,

(2) the exhibiting 2-cell $\omega_{2}^{\mathcal{A}}$ of the left extension $\lambda_{\mathcal{A}}{ }^{5}$ in

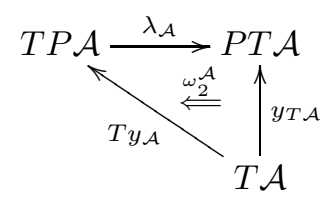

is invertible ${ }^{6}$;

(3) the 1 -cell $\lambda_{\mathcal{A}}$ above is $T_{P}$-cocontinuous ${ }^{7}$;

(4) the respective diagrams
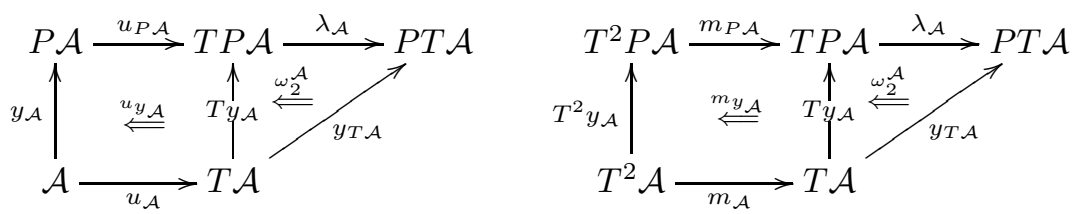

exhibit both $\lambda_{\mathcal{A}} \cdot u_{P \mathcal{A}}$ and $\lambda_{\mathcal{A}} \cdot m_{P \mathcal{A}}$ as left extensions.

\footnotetext{
${ }^{5}$ The left extension is unique up to coherent isomorphism, and exists since $T y_{\mathcal{A}}$ is $P$-admissible.

${ }^{6}$ Equivalently one could ask that each $T y_{\mathcal{A}}$ is $P$-fully faithful [29, Prop. 23].

${ }^{7}$ Equivalently one could ask that each $\lambda_{\mathcal{A}}$ is $T_{P}$-adm-cocontinuous.
} 
Remark 34. Note that a pseudo-distributive law as defined above is unique, as it contains only assertions, and these assertions are invariant under the choice of left left extension (unique up to coherent isomorphism).

3.2. The Main Theorem. We are now ready to state the main result of this section (and this paper), justifying our definitions above.

Theorem 35. Suppose we are given a 2-category $\mathscr{C}$ equipped with a pseudomonad $(T, u, m)$ and a $K Z$ pseudomonad $(P, y, \mu)$. Then the following are equivalent:

(a) $P$ lifts to a $K Z$ doctrine $\widetilde{P}$ on ps-T-alg;

(b) P lifts to a KZ pseudomonad $\widetilde{P}$ on $p s-T$-alg;

(c) P lifts to a pseudomonad $\widetilde{P}$ on ps-T-alg;

(d) There exists a pseudo-distributive law over a KZ doctrine $\lambda: T P \rightarrow P T$;

(e) There exists a pseudo-distributive law over a KZ pseudomonad $\lambda: T P \rightarrow P T$;

(f) There exists a pseudo-distributive law $\lambda: T P \rightarrow P T$.

The proof of this theorem is lengthy, and so we will leave the more difficult aspects of the proof for subsequent subsections. Before moving on to these subsections, we give the remainder of the proof.

Proof of Theorem 35. In order to prove this theorem, we will complete the cycle of implications

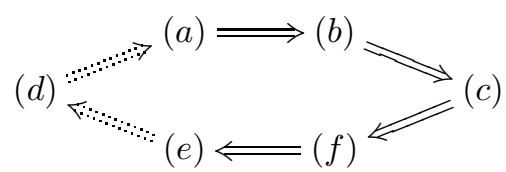

where the more difficult implications left to later sections are dotted above.

$(a) \Longrightarrow(b)$ : A KZ doctrine gives rise to a pseudomonad whose structure forms a fully faithful adjoint string by [25, Theorem 4.1], and this in turn gives rise to a KZ pseudomonad by [21, Prop. 3.1, Lemma 3.2].

$(b) \Longrightarrow(c)$ : This implication is trivial.

$(c) \Longrightarrow(f)$ : For the correspondence between pseudo-distributive laws and liftings to pseudo $T$-algebras see [3, Theorem 5.4].

$(f) \Longrightarrow(e)$ : Given a pseudo-distributive law $\lambda: T P \rightarrow P T$ where $P$ is a $\mathrm{KZ}$ pseudomonad, to check that we then have a pseudo-distributive law over a $\mathrm{KZ}$ pseudomonad in the sense of Definition 29 we need only check the first axiom. But this axiom follows from coherences 7 and 8 as given in [22, Section 4] along with the KZ pseudomonad coherence axiom 2.3.

$(e) \Longrightarrow(d)$ : This is shown later in Theorem 44 .

$(d) \Longrightarrow(a)$ : This is shown later in Theorem 48 .

3.3. Distributive Laws over KZ Monads to those over KZ Doctrines. We will devote this entire subsection to showing that a pseudo-distributive law over a KZ pseudomonad, as in Definition 29, gives rise to a pseudo-distributive law over a KZ doctrine, as in Definition 33. This is $(e) \Longrightarrow(d)$ of Theorem 35. As this is the most difficult implication to show, we will break the proof up into a number of propositions and lemmata, starting with the following proposition.

Note for reader. During this subsection and the next, the reader will keep the three equivalent characterizations of $P$-admissible maps (given in Definition 22) in mind. Indeed, all three characterizations are to be used repeatedly throughout these two subsections.

Remark 36. Most of our diagrams are constructed from the following 2-cells, where $P$ is a $\mathrm{KZ}$ doctrine and $T$ a pseudomonad on a bicategory $\mathscr{C}$ : 
(1) As noted in Definition 22, for any $P$-admissible 1 -cell $L: \mathcal{A} \rightarrow \mathcal{B}$ we have a left extension $\left(R_{L}, \varphi_{L}\right)$ of $y_{\mathcal{A}}$ along $L$. In particular if $L=T y_{\mathcal{A}}$ is $P$ admissible, we will denote this left extension by $\left(\lambda_{\mathcal{A}}, \omega_{2}^{\mathcal{A}}\right)$. Moreover, by $\left[29\right.$, Remark 16], if we are given a chosen right adjoint $\operatorname{res}_{L}$ to $P L$, then the canonical way to define $\left(R_{L}, \varphi_{L}\right)$ is by

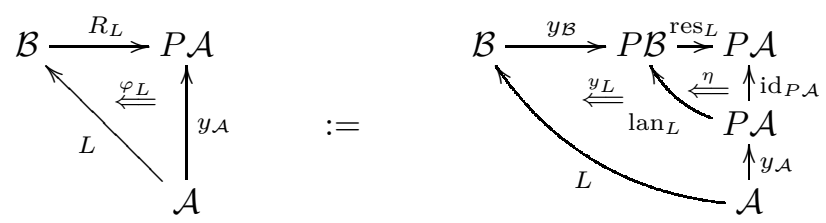

(2) As noted in Definition 18, for any 1-cell $F: \mathcal{A} \rightarrow P \mathcal{B}$ we have a left extension $\left(\bar{F}, c_{F}\right)$ of $F$ along $y_{\mathcal{A}}$ with $c_{F}$ invertible. If $F=R_{L}$ for a $P$-admissible $L$, we will denote this left extension by $\left(\operatorname{res}_{L}, c_{R_{L}}\right)$, and note that $\operatorname{res}_{L}$ defined this way is right adjoint to $P L[29$, Lemma 13].

Proposition 37. Suppose we are given a 2-category $\mathscr{C}$ equipped with a pseudomonad $(T, u, m)$ and a $K Z$ doctrine $(P, y)$. Further suppose that for each object $\mathcal{A} \in \mathscr{C}, T y_{\mathcal{A}}$ is $P$-admissible, and the left extension ${ }^{8}$ which we denote $\lambda_{\mathcal{A}}$ in

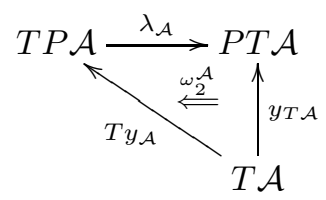

is exhibited by an isomorphism denoted $\omega_{2}^{\mathcal{A}}$. Then for every P-admissible 1-cell $L: \mathcal{A} \rightarrow \mathcal{B}$ such that $T L: T \mathcal{A} \rightarrow T \mathcal{B}$ is also $P$-admissible, the respective pastings
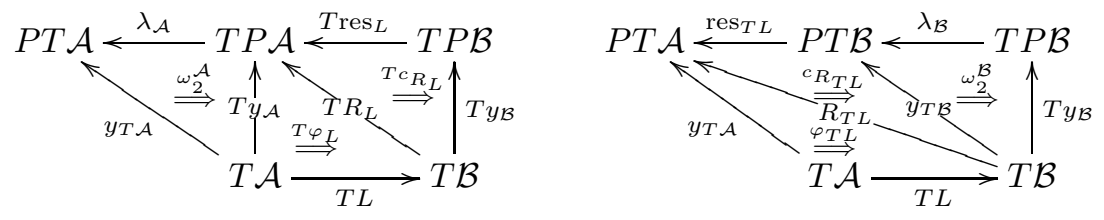

exhibit $\lambda_{\mathcal{A}} \cdot T \operatorname{Tres}_{L}$ and $\operatorname{res}_{T L} \cdot \lambda_{\mathcal{B}}$ as left extensions of $y_{T \mathcal{A}}$ along $T y_{\mathcal{B}} \cdot T L ;$ yielding an isomorphism of left extensions:

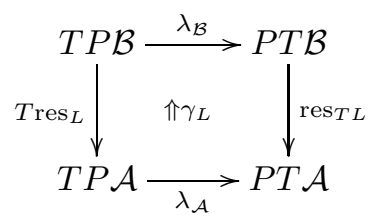

Moreover, if the left diagram below exhibits $R_{L}$ as a left extension
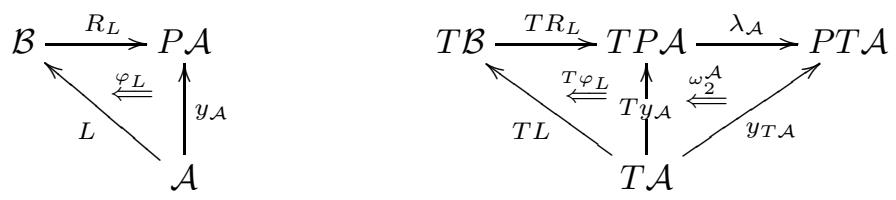

then the right diagram exhibits $\lambda_{\mathcal{A}} \cdot T R_{L}$ as a left extension.

\footnotetext{
${ }^{8}$ This left extension exists since $T y_{\mathcal{A}}$ is $P$-admissible.
} 
Proof. Firstly, we consider the diagram

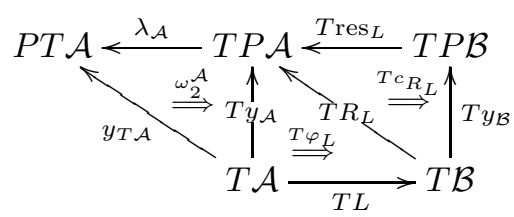

and note that $\lambda_{\mathcal{A}} \cdot \operatorname{Tres}_{L}$ is a left extension since for any 1-cell $H: T P \mathcal{B} \rightarrow P T \mathcal{A}$ we have the natural bijections

$$
\begin{aligned}
\frac{\lambda_{\mathcal{A}} \cdot \operatorname{Tres}_{L}}{\lambda_{\mathcal{A}}} \rightarrow H \\
\hline y_{T \mathcal{A}} \rightarrow H \cdot T \operatorname{lan}_{L} \\
\hline y_{T \mathcal{A}} \rightarrow H \cdot T \operatorname{lan}_{L} \cdot T y_{\mathcal{A}} \cdot T L
\end{aligned}
$$

mates correspondence

since $\lambda_{\mathcal{A}}$ is a left extension

$P L \cdot y_{\mathcal{A}} \cong y_{\mathcal{B}} \cdot L$

and one may check this is the correct exhibiting 2-cell using [29, Remark 16]. We may also consider the diagram

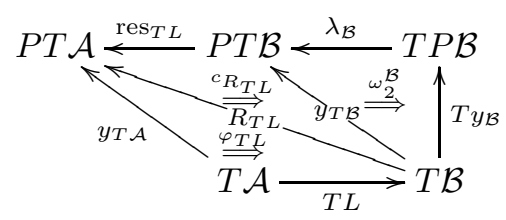

and note that since $T y_{\mathcal{B}}$ is $P$-admissible the left extension $\lambda_{\mathcal{B}}$ is preserved by $\operatorname{res}_{T L}$. Noting $c_{R_{T L}}$ is invertible, we then apply the pasting lemma for left extensions (the dual of [26, Prop. 1]) to see the outside diagram exhibits $\operatorname{res}_{T L} \cdot \lambda_{\mathcal{B}}$ as a left extension. By uniqueness of left extensions, we derive our desired isomorphism $\gamma_{L}: \lambda_{\mathcal{A}} \cdot \operatorname{Tres}_{L} \cong \operatorname{res}_{T L} \cdot \lambda_{\mathcal{B}}$. Now, to show that

$$
T \mathcal{B} \stackrel{T R_{L}}{\longrightarrow} T P \mathcal{A} \stackrel{\lambda_{\mathcal{A}}}{\longrightarrow} P T \mathcal{A}
$$

exhibits $\lambda_{\mathcal{A}} \cdot T R_{L}$ as a left extension, it suffices to show that we have an isomorphism $\lambda_{\mathcal{A}} \cdot T R_{L} \cong R_{T L}$ and that pasting the left extension $\left(R_{T L}, \varphi_{T L}\right)$ with this isomorphism yields the above. This is the case since all regions in the following diagram commute up to isomorphism

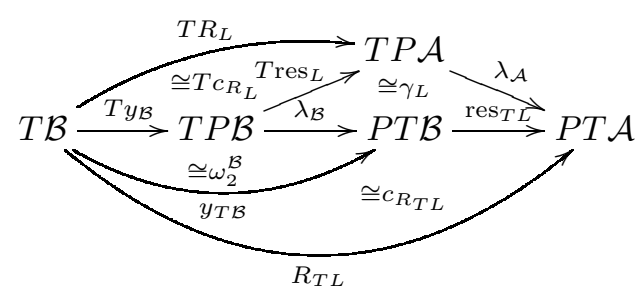

and it is easy to check $\varphi_{T L}$ pasted with this isomorphism yields the pasting 3.2 if one uses the definition of $\gamma_{L}$.

Remark 38. Note that the above proposition tells us something about the components of $\lambda$ being separately cocontinuous, without any assumptions on pseudonaturality of $\lambda$. This may seem unusual in view of the following lemma, in which we show pseudonaturality of $\lambda$ is precisely equivalent to the $T_{P}$-cocontinuity of its components. 
Lemma 39. Suppose we are given a 2-category $\mathscr{C}$ equipped with a pseudomonad $(T, u, m)$ and a $K Z$ doctrine $(P, y)$. Further suppose that for each object $\mathcal{A} \in \mathscr{C}$, $T y_{\mathcal{A}}$ is $P$-admissible and the left extension which we call $\lambda_{\mathcal{A}}$ as on the left below
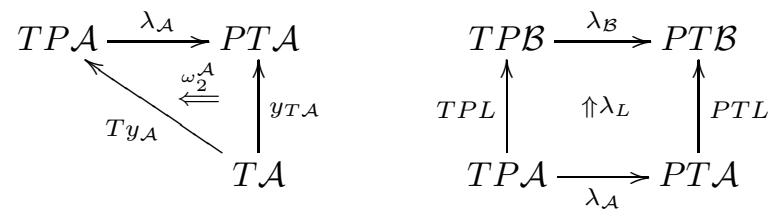

is exhibited by an isomorphism $\omega_{2}^{\mathcal{A}}$. Then for all $L: \mathcal{A} \rightarrow \mathcal{B}$ the naturality squares for $\lambda$ as on the right above commute up to a coherent isomorphism $\lambda_{L}$, with coherent meaning

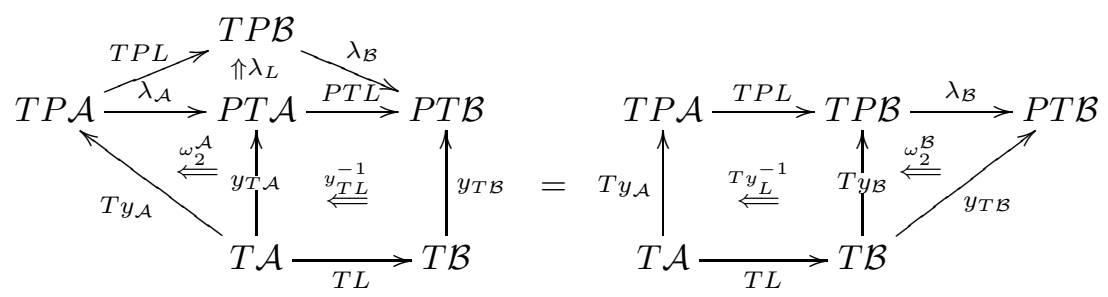

(the condition for $\omega_{2}$ to be a modification), if and only if each $\lambda_{\mathcal{A}}$ is $T_{P}$-cocontinuous.

Proof. The following implications prove the logical equivalence.

$(\Longrightarrow)$ : Suppose that for each $L: \mathcal{A} \rightarrow \mathcal{B}$ the naturality square of $\lambda$ commutes up to a coherent isomorphism $\lambda_{L}$. Then noting that $\operatorname{id}_{P \mathcal{B}}=R_{y_{\mathcal{B}}}$, we see that for any left extension as on the left (which is isomorphic to $\left(\bar{F}, c_{F}\right)$ by uniqueness)
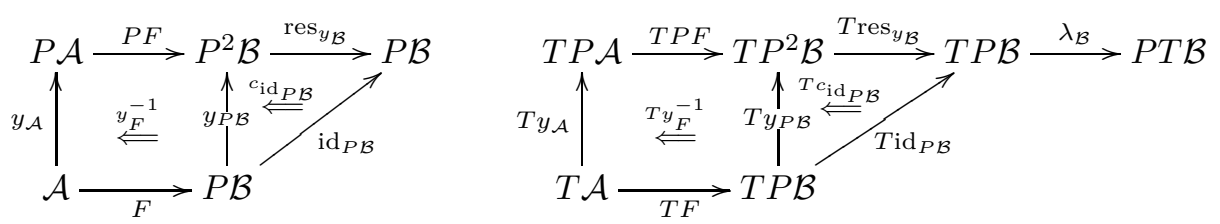

it suffices to check that the right diagram above exhibits $\lambda_{\mathcal{B}} \cdot T \operatorname{res}_{y_{\mathcal{B}}} \cdot T P F$ as a left extension. To see this we note that the pasting

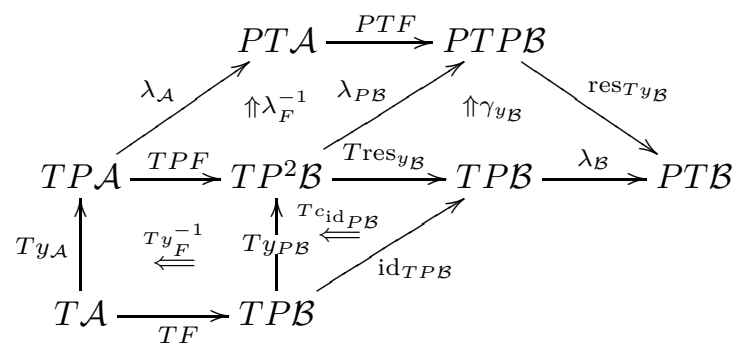

is equal to the pasting (using $\lambda_{\mathcal{B}}=R_{T y_{\mathcal{B}}}$ )

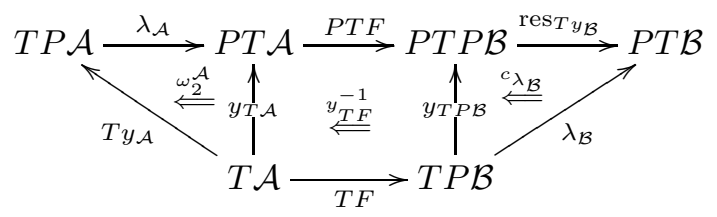

This is shown by first using the coherence condition on $\lambda_{F}^{-1}$, and then using the definition of $\gamma_{y_{\mathcal{B}}}$ from Proposition 37. Note also this last diagram exhibits $\operatorname{res}_{T y_{\mathcal{B}}}$. 
$P T F \cdot \lambda_{\mathcal{A}}$ as a left extension since $T y_{\mathcal{A}}$ is $P$-admissible (using preservation of the left extension $\lambda_{\mathcal{A}}$ by $P$-homomorphisms).

$(\Longleftarrow)$ : For any $L: \mathcal{A} \rightarrow \mathcal{B}$, we know $P T L \cdot \lambda_{\mathcal{A}}$ is a left extension of $y_{T \mathcal{B}} \cdot T L$ along $T y_{\mathcal{A}}$ since $T y_{\mathcal{A}}$ is $P$-admissible. Also $\lambda_{\mathcal{B}} \cdot T P L$ is such a left extension as $\lambda_{\mathcal{B}}$ is $T_{P}$-cocontinuous, giving us an isomorphism of left extensions $\lambda_{F}$ coherent as in the statement of this lemma.

Remark 40. A Beck condition is satisfied here. Indeed, the 2-cell $\gamma_{L}$ as in Proposition 37 is the mate of $\lambda_{L}$ as in the above lemma. This may be seen by pasting the left diagram of 3.1 with the mate of $\lambda_{L}$ and then recovering the right diagram (making use of [29, Remark 16] and the coherence condition on $\lambda_{L}$ ).

In the following lemma we see that for a pseudo-distributive law over a KZ pseudomonad as in Definition 29, the modification components $\omega_{2}^{\mathcal{A}}$ necessarily exhibit each $\lambda_{\mathcal{A}}$ as a left extension, and from this we deduce the existence of invertible components $\omega_{4}^{\mathcal{A}}$.

Lemma 41. Suppose we are given a 2-category $\mathscr{C}$ equipped with a pseudomonad $(T, u, m)$ and a $K Z$ pseudomonad $(P, y, \mu)$. Suppose further that we are given a pseudo-distributive law over a KZ pseudomonad $\lambda: T P \rightarrow P T$. Then for each $\mathcal{A} \in \mathscr{C}, T y_{\mathcal{A}}$ is $P$-admissible, exhibited by an adjunction

$$
P T y_{\mathcal{A}} \dashv \mu_{T \mathcal{A}} \cdot P \lambda_{\mathcal{A}}
$$

Moreover, the diagrams as on the left exhibit each $\lambda_{\mathcal{A}}$ as a left extension,
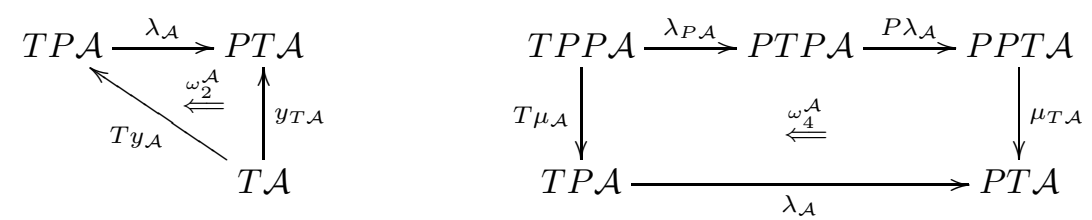

and there exists canonical isomorphisms as on the right for each $\mathcal{A}$.

Proof. We now prove the three assertions of the above lemma.

EACH $T y_{\mathcal{A}}$ IS $P$-ADMissible. Firstly, we note that the below diagram exhibits $\mu_{T \mathcal{A}} \cdot P \lambda_{\mathcal{A}}$ as a left extension

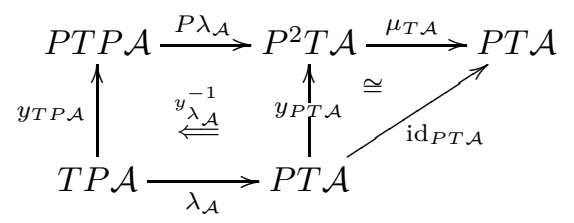

Indeed, the construction of a $\mathrm{KZ}$ doctrine from a pseudomonad whose structure forms a fully faithful adjoint string is outlined in [25], and the above is an instance of this construction. Noting $\overline{\lambda_{\mathcal{A}}}=\mu_{T \mathcal{A}} \cdot P \lambda_{\mathcal{A}}$, we define our unit $\eta$ as the unique solution to the left extension problem

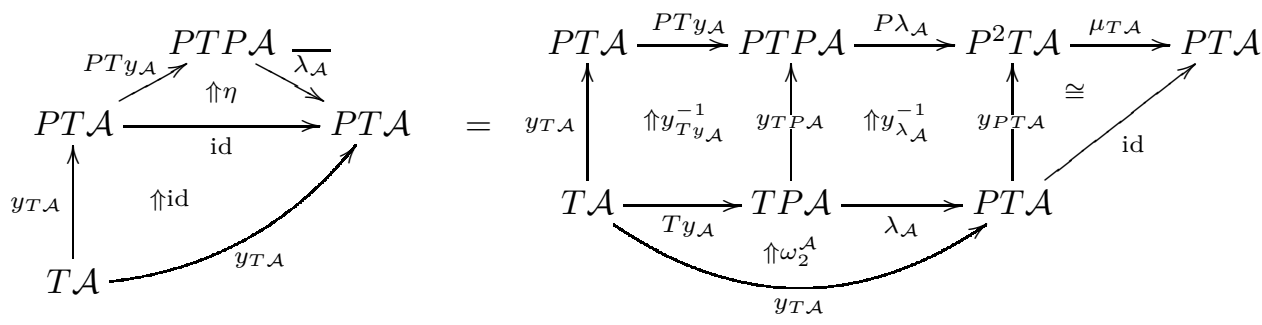


Note that the unit $\eta$ must then be given by

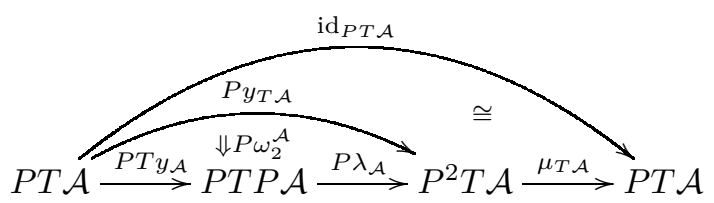

as $y: 1 \rightarrow P$ is a pseudonatural transformation. We define our counit $\varepsilon$ as the unique solution to the left extension problem
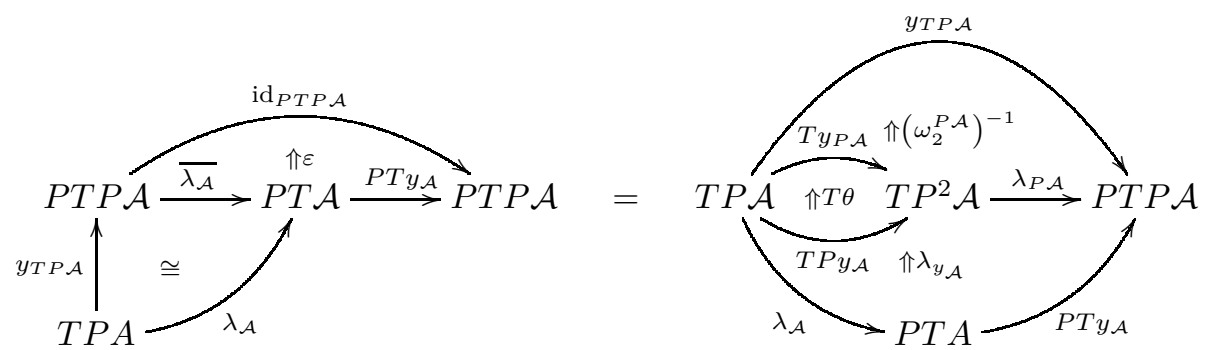

where the unnamed isomorphism above is 3.3. One could also define $\varepsilon$ directly in terms of $\theta$, but that would result in a more complicated proof. Of the triangle identities:
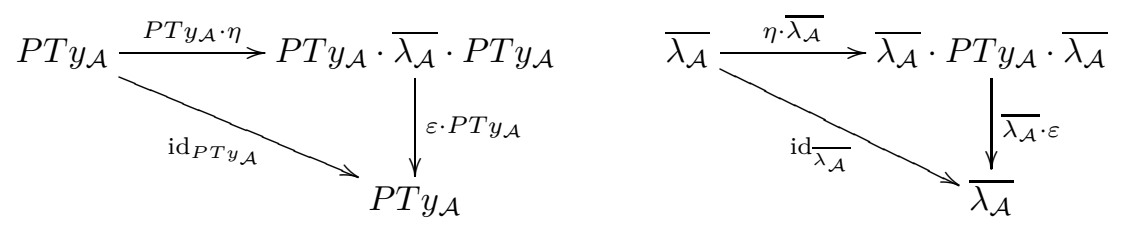

the left identity, which is equivalent to asking for equality when whiskered by $y_{T \mathcal{A}}$, can be proven using that $\omega_{2}$ is a modification. The right triangle identity, which is equivalent to asking for equality when whiskered by $y_{T P \mathcal{A}}$, amounts to asking that the pasting

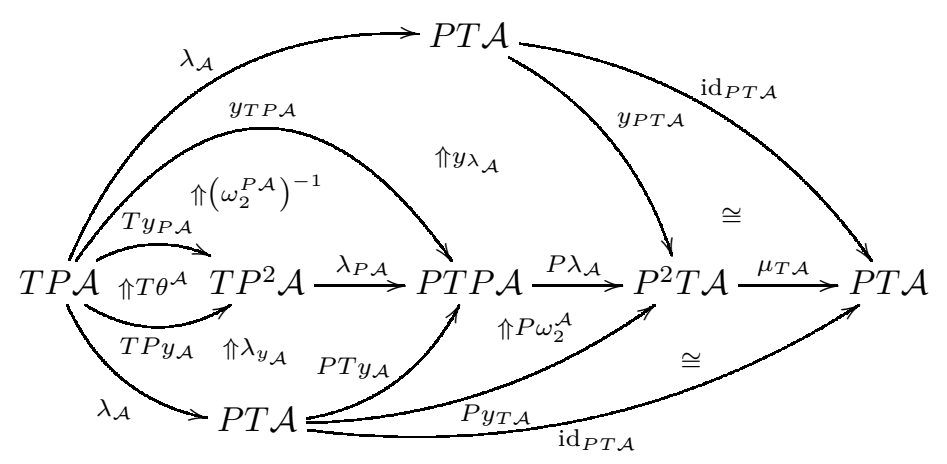

is the identity. This is where the first axiom for a pseudo-distributive law over a $\mathrm{KZ}$ pseudomonad is used, in addition to the second coherence axiom 2.3 of a $\mathrm{KZ}$ pseudomonad. 
EACH $\omega_{2}^{\mathcal{A}}$ Exhibits $\lambda_{\mathcal{A}}$ As A Left extension. As $T y_{\mathcal{A}}$ is $P$-admissible, we know by [29, Remark 16] that the pasting

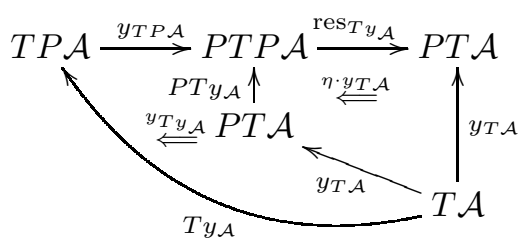

exhibits $\operatorname{res}_{T y_{\mathcal{A}}} \cdot y_{T P \mathcal{A}}$ as a left extension, where $\operatorname{res}_{T y_{\mathcal{A}}}=\overline{\lambda_{\mathcal{A}}}=\mu_{T \mathcal{A}} \cdot P \lambda_{\mathcal{A}}$, and $\eta$ is the unit of $P T y_{\mathcal{A}} \dashv \operatorname{res}_{T y_{\mathcal{A}}}$ as just defined. From a substitution of the definition of $\eta$ (and pasting with a couple of isomorphisms) we see that the pasting

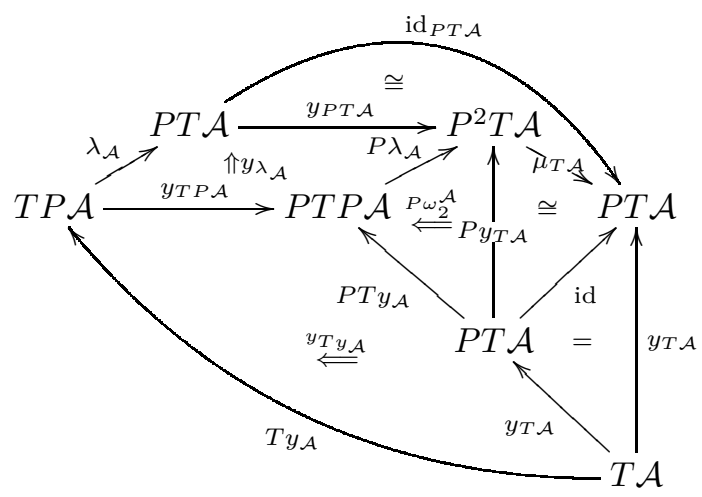

exhibits $\lambda_{\mathcal{A}}$ as a left extension. Note that this pasting is equal to $\omega_{2}$ as a consequence of $\omega_{2}$ being a modification as well as the coherence condition 2.1 satisfied by $P$.

THERE EXISTS CANONICAL ISOMORPHISMS $\omega_{4}^{\mathcal{A}}$. We have the left extension

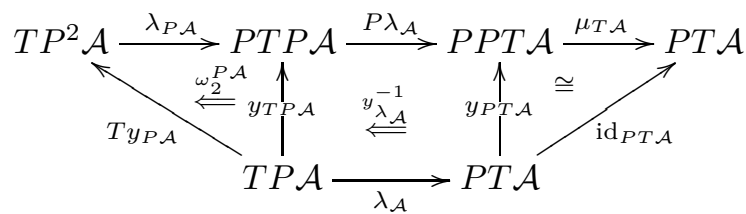

since $T y_{P \mathcal{A}}$ is $P$-admissible, and also the left extension

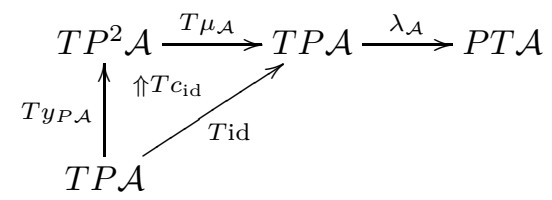

since $\lambda_{\mathcal{A}}$ is $T_{P}$-cocontinuous by Lemma 39 , giving us our isomorphism of left extensions $\omega_{4}^{\mathcal{A}}$. Note that this means $\omega_{4}$ satisfies coherence axiom 7 of [22].

In the following proposition we show that the admissible maps are preserved. Note that the proof relies on the existence of isomorphisms $\omega_{4}^{\mathcal{A}}$ as above, which in turn relies on the the admissibility of $y_{\mathcal{A}}$ being preserved (also shown above).

Proposition 42. Suppose we are given a 2-category $\mathscr{C}$ equipped with a pseudomonad $(T, u, m)$ and a $K Z$ pseudomonad $(P, y, \mu)$. Suppose further that we are given a pseudo-distributive law over a KZ pseudomonad $\lambda: T P \rightarrow P T$. Then $T$ preserves $P$-admissible maps.

Proof. Suppose we are given a 1 -cell $L: \mathcal{A} \rightarrow \mathcal{B}$ which is $P$-admissible, meaning that we have an adjunction $P L \dashv \operatorname{res}_{L}$ with unit and counit denoted $\eta$ and $\varepsilon$ respectively. 
We show that $T L: T \mathcal{A} \rightarrow T \mathcal{B}$ must then be $P$-admissible, with the admissibility exhibited by an adjunction

$$
P T L \dashv \mu_{T \mathcal{A}} \cdot P \lambda_{\mathcal{A}} \cdot P \operatorname{Pres}_{L} \cdot P T y_{\mathcal{B}}
$$

Firstly, we note that this right adjoint is exhibited as the left extension

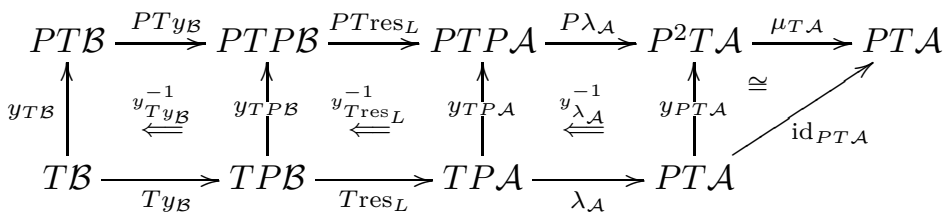

and denote it $\mathbf{R}_{L}$ for convenience. We then define our unit $n$ as the unique 2-cell rendering

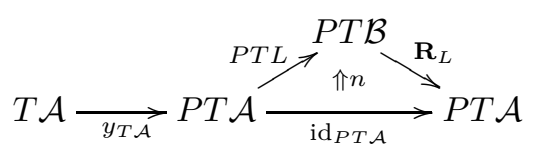

equal to

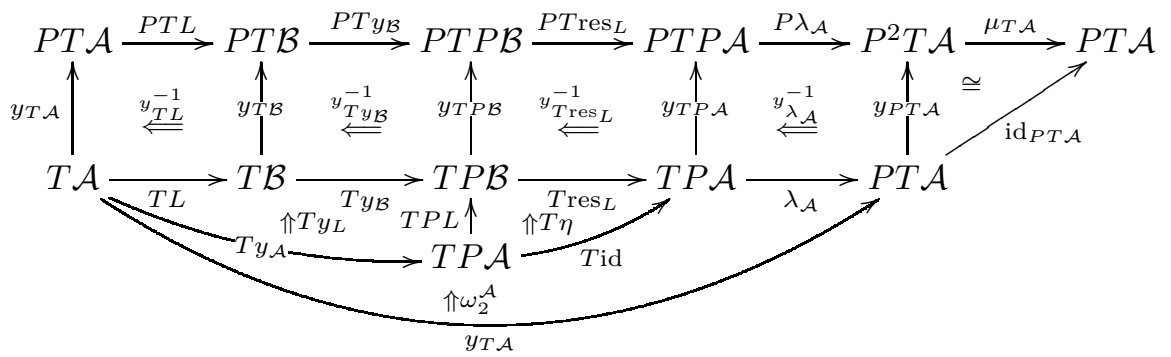

and note that the unit $n$ is then given by

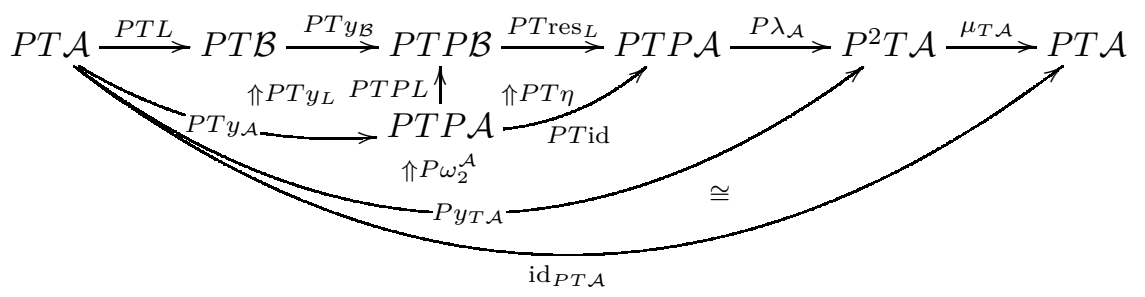

as $y: 1 \rightarrow P$ is a pseudonatural transformation. We define our counit $e$ as the unique solution to the left extension problem

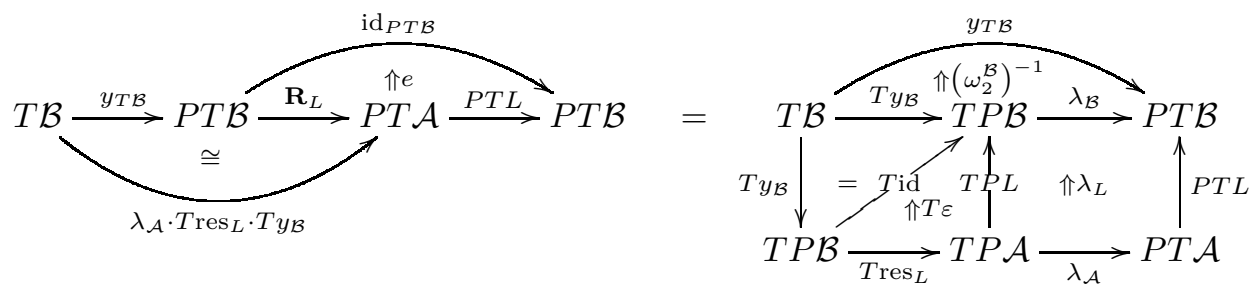

where the unlabeled isomorphism is 3.4. Of the triangle identities:
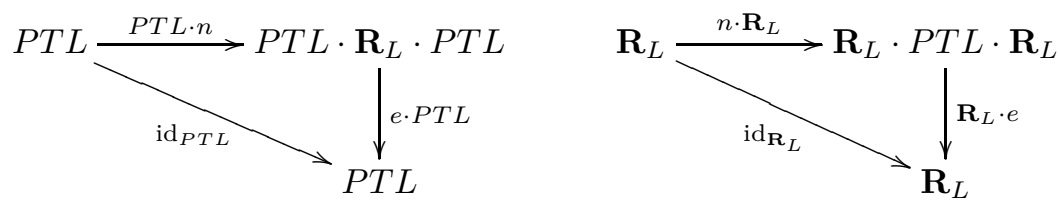
the left identity (or equivalently the left triangle identity whiskered by $y_{T \mathcal{A}}$ ) easily follows from the whiskered definitions of $n$ and $e$ as well as the corresponding triangle identity for $P L \dashv \operatorname{res}_{L}$, and $\omega_{2}$ being a modification. The right triangle identity (or that whiskered by $y_{T \mathcal{B}}$ ) is more complicated. This identity amounts to checking that

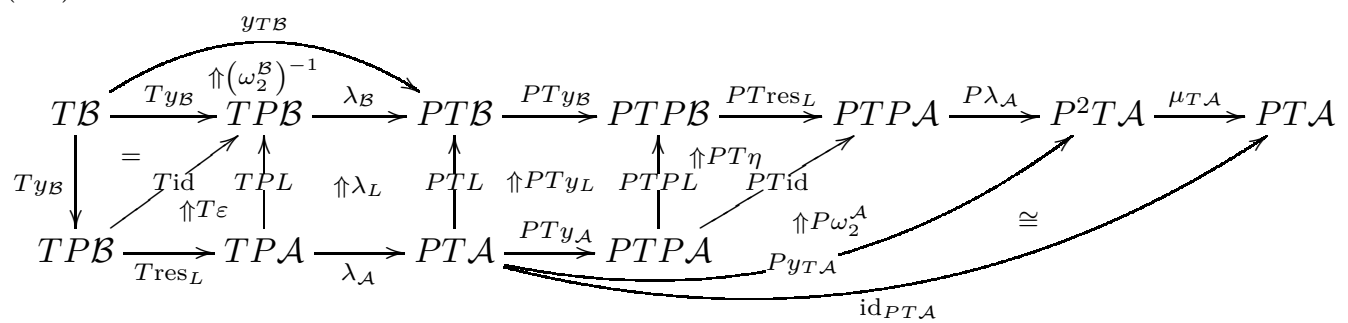

is just the isomorphism 3.4. The first step here is to make our diagrams more like the first axiom of a pseudo-distributive law over a $\mathrm{KZ}$ doctrine. Upon using that $\omega_{2}$ is a modification and the coherence axiom 2.3, the problem reduces to showing that 3.6 with the unnamed isomorphism and cell $\omega_{2}^{\mathcal{B}}$ removed is equal to

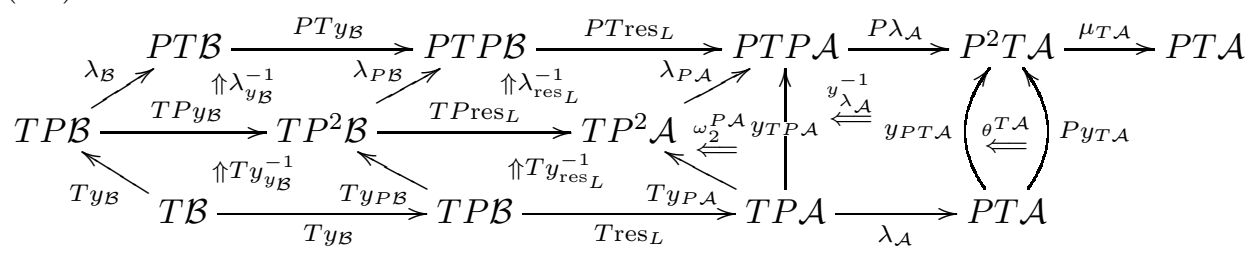

We then simplify 3.7 using the first axiom of a pseudo-distributive law over a $\mathrm{KZ}$ doctrine, canceling $P \omega_{2}^{\mathcal{A}}$, and pasting $\lambda_{y_{\mathcal{B}}}, \lambda_{y_{\mathcal{A}}}$ and $\lambda_{\operatorname{res}_{L}}$ to the other side of the desired equation. By pseudonaturality of $\lambda$, the problem may then be reduced to showing that

$$
T \mathcal{B} \stackrel{T y_{\mathcal{B}}}{\longrightarrow} T P \mathcal{B} \stackrel{T P y_{\mathcal{B}}}{\longrightarrow} T P^{2} \mathcal{B} \stackrel{T P \operatorname{res}_{L}}{\longrightarrow} T P^{2} \mathcal{A} \stackrel{\lambda_{P \mathcal{A}}}{\longrightarrow} P T P \mathcal{A} \stackrel{P \lambda_{\mathcal{A}}}{\longrightarrow} P^{2} T \mathcal{A} \stackrel{\mu_{T \mathcal{A}}}{\longrightarrow} P T \mathcal{A}
$$

is equal to

$$
\begin{aligned}
& T P \mathcal{B} \stackrel{T P y_{\mathcal{B}}}{\longrightarrow} T P^{2} \mathcal{B} \stackrel{T P \operatorname{Pres}_{L}}{\longrightarrow} T P^{2} \mathcal{A} \stackrel{\lambda_{P \mathcal{A}}}{\longrightarrow} P T P \mathcal{A} \stackrel{P \lambda_{\mathcal{A}}}{\longrightarrow} P^{2} T \mathcal{A} \stackrel{\mu_{T \mathcal{A}}}{\longrightarrow} P T \mathcal{A}
\end{aligned}
$$

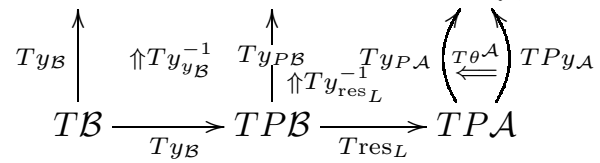

Since we have the isomorphism $\omega_{4}^{\mathcal{A}}$ as in Lemma 41 , and as $T \theta^{\mathcal{B}} \cdot T y_{\mathcal{B}}$ is invertible (meaning we can paste with $T \theta^{\mathcal{B}}$ and maintain the logical equivalence), we may reduce the problem to showing that

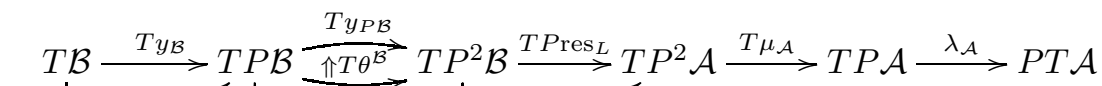

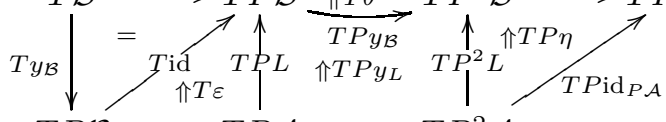

$$
\begin{aligned}
& T P \mathcal{B} \underset{T \operatorname{res}_{L}}{\longrightarrow} T P \mathcal{A} \underset{T P y_{\mathcal{A}}}{\longrightarrow} T P^{2} \mathcal{A}
\end{aligned}
$$


is equal to

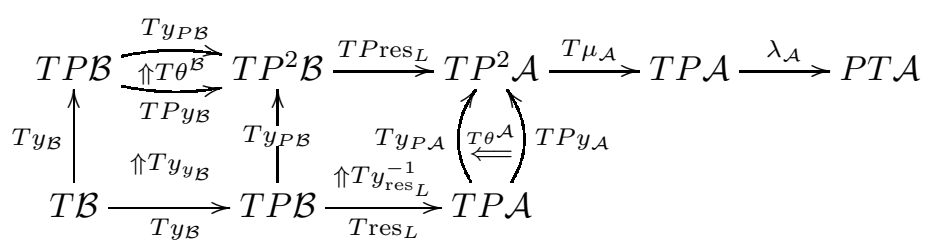

From here, use that $\theta$ is a modification, the axioms 2.2 and 2.3, and pseudonaturality of $y$ to deduce the triangle identity from that of the adjunction $P L \dashv \operatorname{res}_{L}$.

Remark 43. Note that here, as well as in the preceding lemma, we only used that $\omega_{2}$ is an invertible modification and the first axiom for a pseudo-distributive law over a KZ doctrine, along with pseudo naturality of $\lambda$.

We are now ready to prove the main result of this subsection.

Theorem 44. In the statement of Theorem 35 (e) implies (d).

Proof. We first note by Proposition 42 that $T$ preserves $P$-admissible maps. Also, we know by Lemma 41 that each $\lambda_{\mathcal{A}}$ is a left extension exhibited by the distributive law data as in

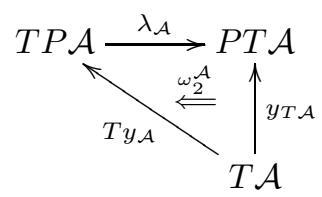

with $\omega_{2}^{\mathcal{A}}$ invertible by assumption. That each $\lambda_{\mathcal{A}}$ is $T_{P}$-cocontinuous is a consequence of Lemma 39 and $\omega_{2}$ being a modification. Finally, that the diagrams
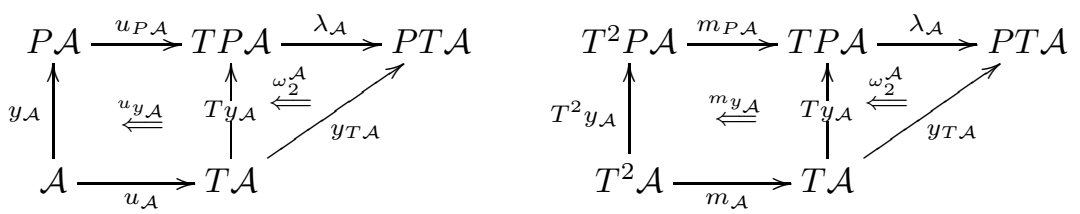

exhibit both $\lambda_{\mathcal{A}} \cdot u_{P \mathcal{A}}$ and $\lambda_{\mathcal{A}} \cdot m_{P \mathcal{A}}$ as left extensions is due to the last two axioms for a pseudo-distributive law over a $\mathrm{KZ}$ pseudomonad (as pasting a left extension with an isomorphism $\omega_{1}$ or $\omega_{3}$ will preserve the left extension property). Indeed, it is clear the left diagram below exhibits $P u_{\mathcal{A}}$ as a left extension.
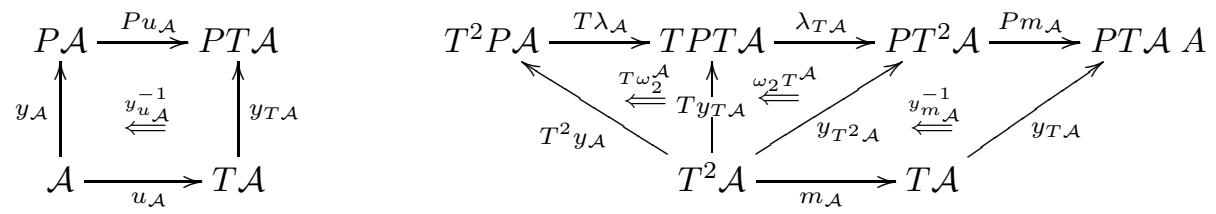

To see that the composite $P m_{\mathcal{A}} \cdot \lambda_{T \mathcal{A}} \cdot T \lambda_{\mathcal{A}}$ on the right is a left extension, note that Proposition 37 shows $\lambda_{T \mathcal{A}} \cdot T \lambda_{\mathcal{A}}$ is a left extension above, and since $T^{2} y_{\mathcal{A}}$ is $P$-admissible by Proposition 42 , the left extension property is respected upon whiskering by $P m_{\mathcal{A}}$.

3.4. Lifting a KZ Doctrine to Algebras via a Distributive Law. In this subsection we show that given a pseudo-distributive law of a pseudomonad $T$ over a KZ doctrine $P$, we may lift $P$ to a $\mathrm{KZ}$ doctrine $\widetilde{P}$ on the 2 -category of pseudo $T$-algebras. This is $(d) \Longrightarrow(a)$ of Theorem 35 . However, before we show this implication we will first need to verify the following proposition. 
Proposition 45. Suppose we are given statement $(d)$ of Theorem 35 . It then follows that:

(1) T preserves $P$-admissible maps;

and for every pseudo $T$-algebra $(\mathcal{A}, T \mathcal{A} \stackrel{x}{\rightarrow} \mathcal{A})$,

(2) there exists a 1-cell $z_{x}$ given as the left extension via an isomorphism $\xi_{x}$

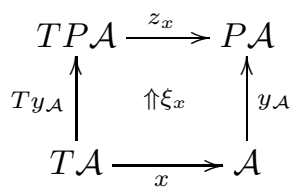

which we call the Day convolution at $x$;

(3) each $z_{x}$ is $T_{P}$-cocontinuous;

(4) the respective diagrams
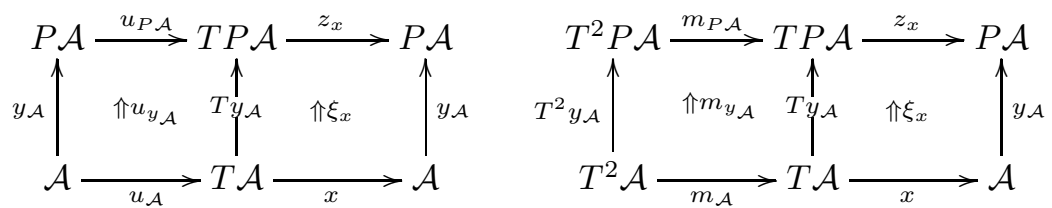

exhibit $z_{x} \cdot u_{P \mathcal{A}}$ and $z_{x} \cdot m_{P \mathcal{A}}$ as left extensions.

Proof. (1) This property is straight from the definition. We include this property here so that this proposition may be taken as one the equivalent conditions of Theorem 35. We will remark about this later in this subsection. Now, let a pseudo $T$-algebra $(\mathcal{A}, T \mathcal{A} \stackrel{x}{\rightarrow} \mathcal{A})$ be given. (2) The left extension $\left(z_{x}, \xi_{x}\right)$ is given by the diagram

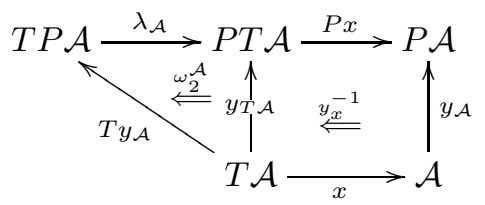

where the left extension $\lambda_{\mathcal{A}}$ is preserved by $P x$ as $T y_{\mathcal{A}}$ is $P$-admissible. (3) Suppose we are given a left extension as on the left below.
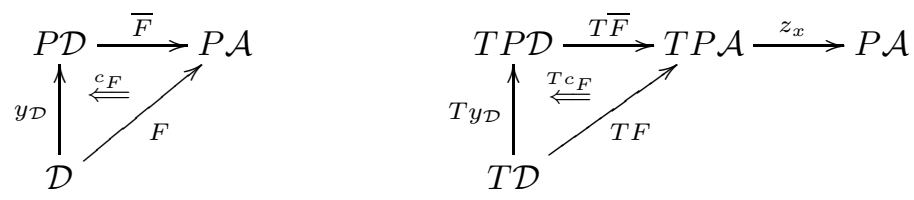

As this left extension is $T$-preserved by $\lambda_{\mathcal{A}}$, which in turn is preserved by $P x$ as $T y_{\mathcal{D}}$ is $P$-admissible, the diagram on the right exhibits $z_{x} \cdot T \bar{F}=P x \cdot \lambda_{\mathcal{A}} \cdot T \bar{F}$ as a left extension. (4) Again noting each $T y_{\mathcal{A}}$ is $P$-admissible, we see the left extensions
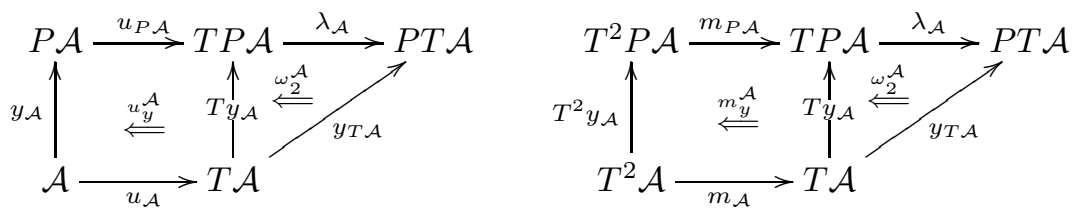

are preserved upon composing with $P x$. Trivially, these left extensions are then preserved upon pasting with the isomorphism $y_{x}$. 
The following remark is not needed for the proof of Theorem 35, it merely explains why the consequences in the above proposition are equivalent to the conditions $(a)$ through to $(f)$ of this theorem.

Remark 46. Note that from this proposition one may recover statement (d) of Theorem 35. This is since given the data of this proposition, one may recover a choice of each $\lambda_{\mathcal{A}}$ and its exhibiting invertible 2-cell $\omega_{2}^{\mathcal{A}}$ as a left extension, by taking the pasting

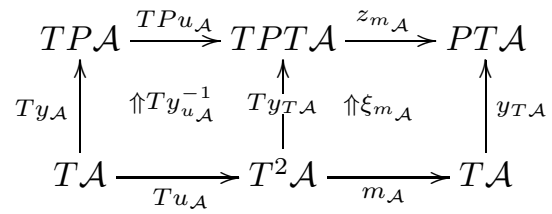

The condition of each $\lambda_{\mathcal{A}}$ being $T_{P^{-c o c o n t i n u o u s ~}}$ is inherited from the corresponding condition on each $z_{m_{\mathcal{A}}}$. Condition (4) of this proposition yields the corresponding conditions on the maps $\lambda_{\mathcal{A}}$. We omit this last calculation, as it is not required for the proof of the main theorem. We just note that this last calculation relies on the pseudo-algebra structure of the maps $z_{x}: T P \mathcal{A} \rightarrow P \mathcal{A}$ constructed later on in this subsection. The construction of the algebra structure may be done with all of the axioms for a pseudo-distributive law over a $\mathrm{KZ}$ doctrine without the last (which we have recovered from the proposition), in addition to the last condition of the proposition.

The following proposition will be useful in the proof that $(d)$ implies $(a)$.

Proposition 47. Suppose we are given a 2-category $\mathscr{C}$ equipped with a pseudomonad $(T, u, m)$ and a $K Z$ doctrine $(P, y)$. Further suppose that we are given a pseudo-distributive law over a KZ doctrine $\lambda: T P \rightarrow P T$. Then for any two $P$-cocomplete objects $\mathcal{C}$ and $\mathcal{D}$, a 1-cell $u: T \mathcal{C} \rightarrow \mathcal{D}$ is $T_{P}$-cocontinuous if and only if it is $T_{P}$-adm-cocontinuous.

Proof. Supposing that $u$ is $T_{P}$-cocontinuous we check that $u$ is necessarily $T_{P}$-admcocontinuous. To see this, we first note that we have an induced isomorphism of left extensions as a consequence of having the two left extensions
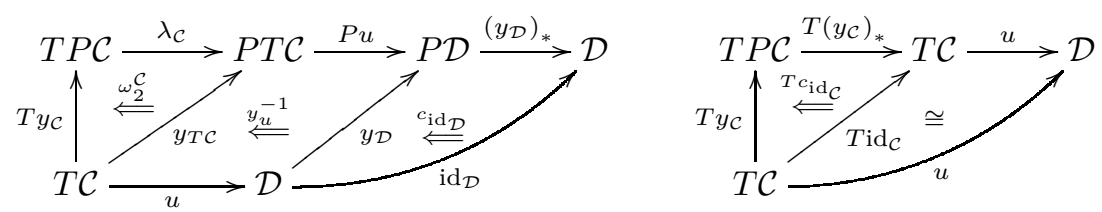

We must check that the left extension (where $L$ is $P$-admissible)

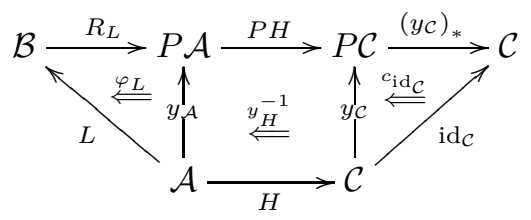

is $T$-preserved by $u$. Indeed, on applying $T$ and whiskering by $u$, and then pasting with this isomorphism of left extensions and a naturality isomorphism of $\lambda$ (which 
we have by Lemma 39), we obtain

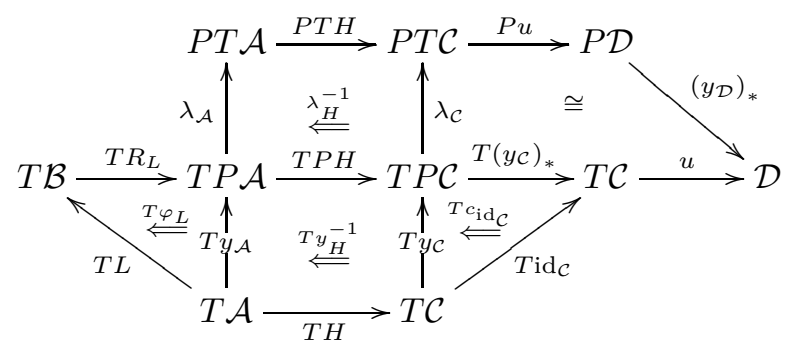

Then noting that pasting with invertible 2-cells preserves left extensions and that

$$
T \mathcal{B} \stackrel{T R_{L}}{\longrightarrow} T P \mathcal{A} \stackrel{\lambda_{\mathcal{A}}}{\longrightarrow} P T \mathcal{A} \stackrel{P T H}{\longrightarrow} P T \mathcal{C} \stackrel{P u}{\longrightarrow} P \mathcal{P} \stackrel{\left(y_{\mathcal{D}}\right)_{*}}{\longrightarrow} \mathcal{D}
$$

is a left extension as a consequence of $T L$ being $P$-admissible (thus the left extension $\lambda_{\mathcal{A}} \cdot T R_{L}$ in Proposition 37 being preserved), we have the result.

We now have everything required to complete the proof of the main theorem.

Theorem 48. In the statement of Theorem 35 (d) implies (a).

Proof. Firstly, we observe that each $z_{x}$ is $T_{P}$-adm-cocontinuous as a consequence of Proposition 47. It follows that we have the left extensions

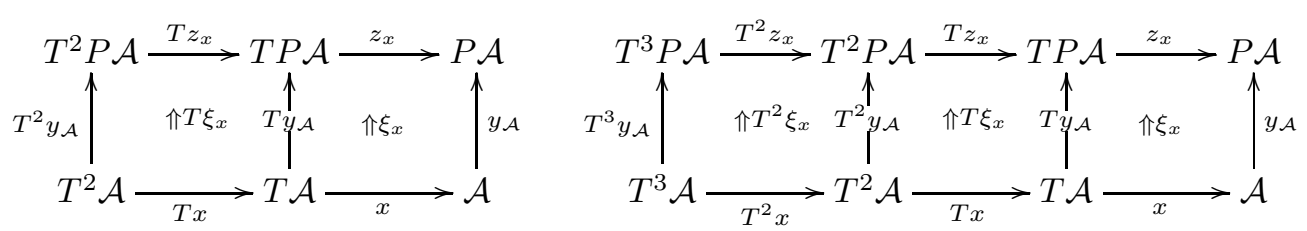

upon noting that each $T^{2} y_{\mathcal{A}}$ and $T^{3} y_{\mathcal{A}}$ is $P$-admissible.

Secondly, we check that each $\left(P \mathcal{A}, z_{x}\right)$ is a pseudo $T$-algebra. We define our algebra structure maps as the unique solutions to the following left extension problems (and note they are invertible as they are isomorphisms of left extensions by Proposition 45)
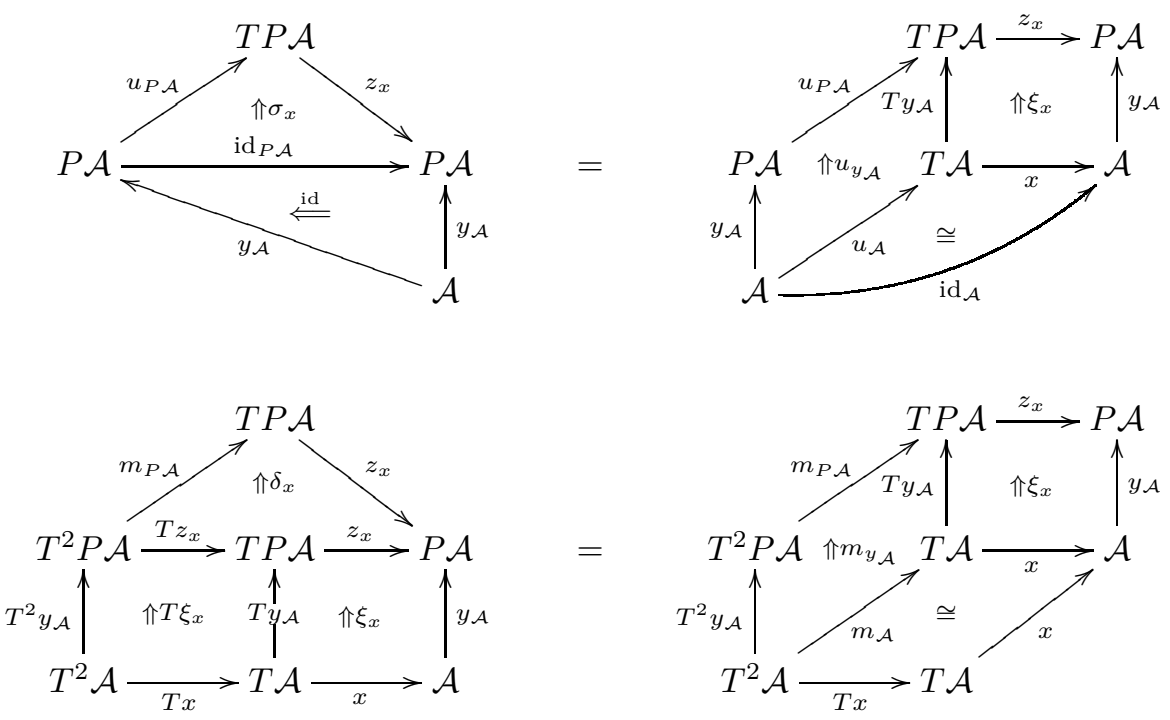
Note that these are the axioms for $\xi_{x}$ to exhibit $y_{\mathcal{A}}$ as a pseudo $T$-morphism. To check that the algebra structure coherence axioms are satisfied, we note that the equalities

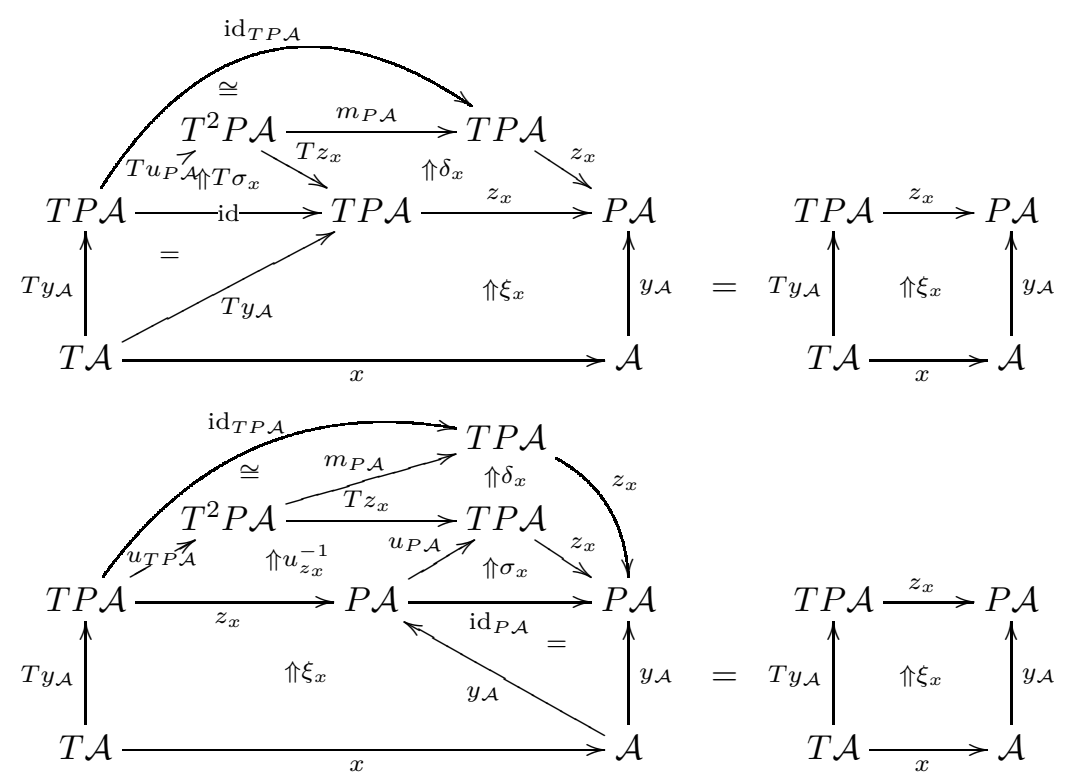

and the equality between

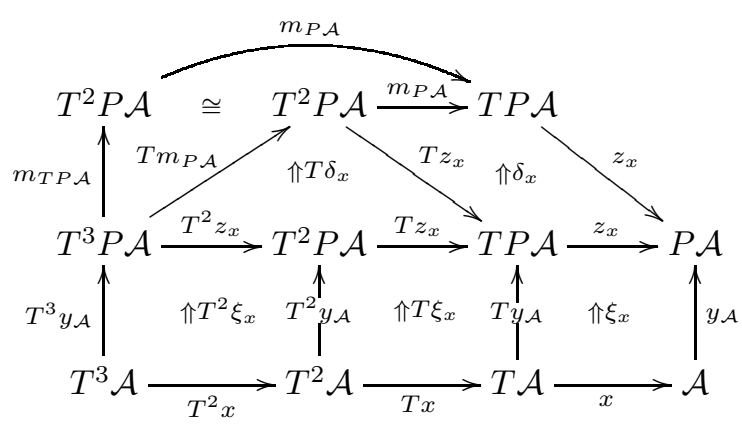

and

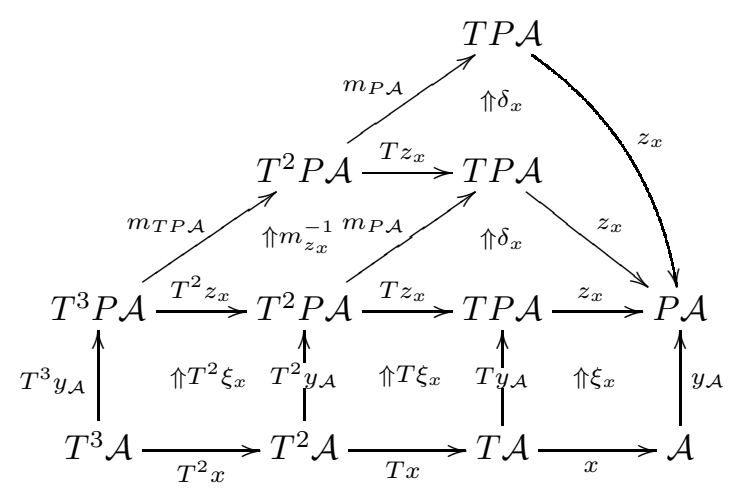

easily follow from the respective conditions on $(\mathcal{A}, x)$ being a pseudo $T$-algebra and the definitions of $\delta_{x}$ and $\sigma_{x}$.

We now use the above to define our KZ doctrine

$$
\widetilde{P}: \text { ps- } T \text {-alg } \rightarrow \text { ps- } T \text {-alg }
$$


We use the assignment on objects $(\mathcal{A}, x) \mapsto\left(P \mathcal{A}, z_{x}\right)$. We take our units as the pseudo $T$-morphisms $\left(y_{\mathcal{A}}, \xi_{x}\right):(\mathcal{A}, x) \rightarrow\left(P \mathcal{A}, z_{x}\right)$. Now suppose that we are given a pseudo $T$-morphism $(F, \phi):(\mathcal{A}, x) \rightarrow\left(P \mathcal{B}, z_{r}\right)$, where $\left(P \mathcal{B}, z_{r}\right)=\widetilde{P}(\mathcal{B}, r)$, as in the diagram

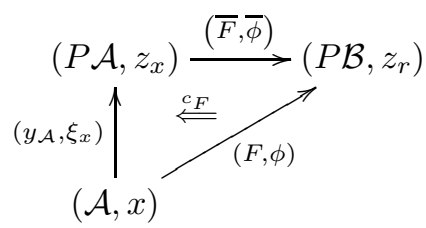

Since $z_{r}$ is $T_{P}$-cocontinuous, we may apply Proposition 14 to find a lax $T$-morphism $(\bar{F}, \bar{\phi})$ as above. Indeed the lax structure map $\bar{\phi}$ is given as the unique solution to

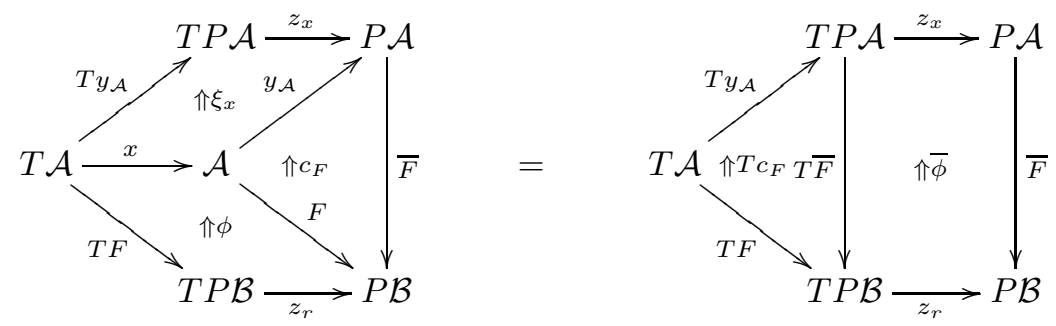

But we notice that
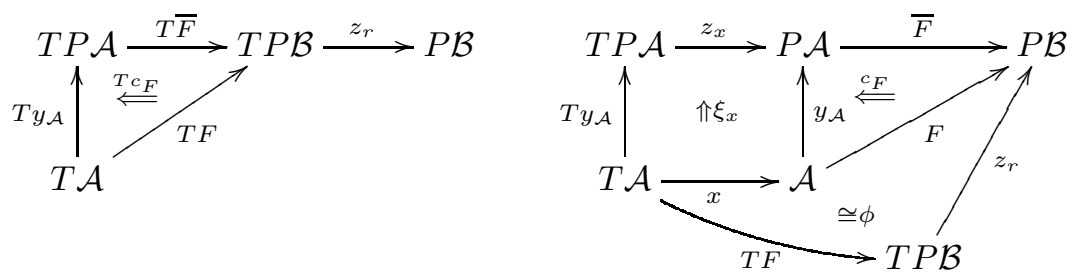

are both left extensions since $z_{r}$ is $T_{P}$-cocontinuous and $T y_{\mathcal{A}}$ is $P$-admissible respectively. It follows that the lax $T$-morphism structure map $\bar{\phi}$ is an isomorphism of left extensions, making $(\bar{F}, \bar{\phi})$ a pseudo $T$-morphism. Of course, if we only assume $(F, \phi)$ to be a lax $T$-morphism then we can only expect $\bar{F}$ to admit a lax $T$-morphism structure.

We now check that such left extensions are preserved by other left extensions of this form. Suppose we are given two left extensions of pseudo $T$-algebras and pseudo $T$-morphisms

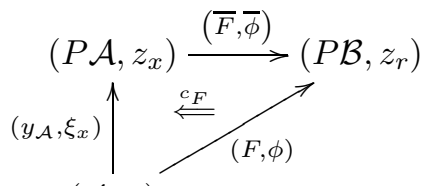

$(\mathcal{A}, x)$

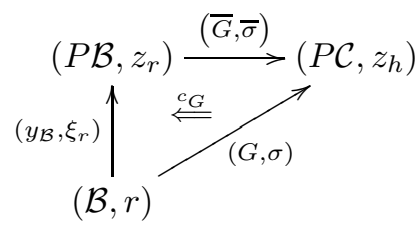

$(\mathcal{B}, r)$

To see that

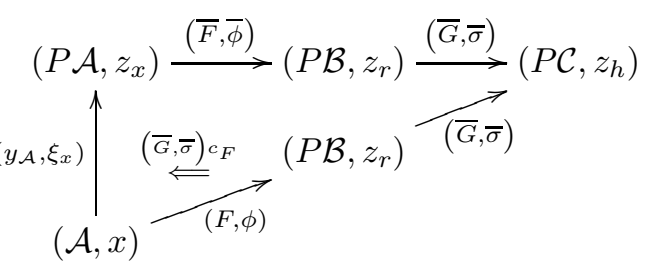

is a left extension we need only observe that the $T$-morphism structure on $\overline{G F}$ resulting from an application of Proposition 14 (on the outside diagram) is given 
by composing $\bar{\phi}$ and $\bar{\sigma}$ as above. This is shown by pasting the defining diagram for $\bar{\phi}$ with $\bar{\sigma}$ which gives

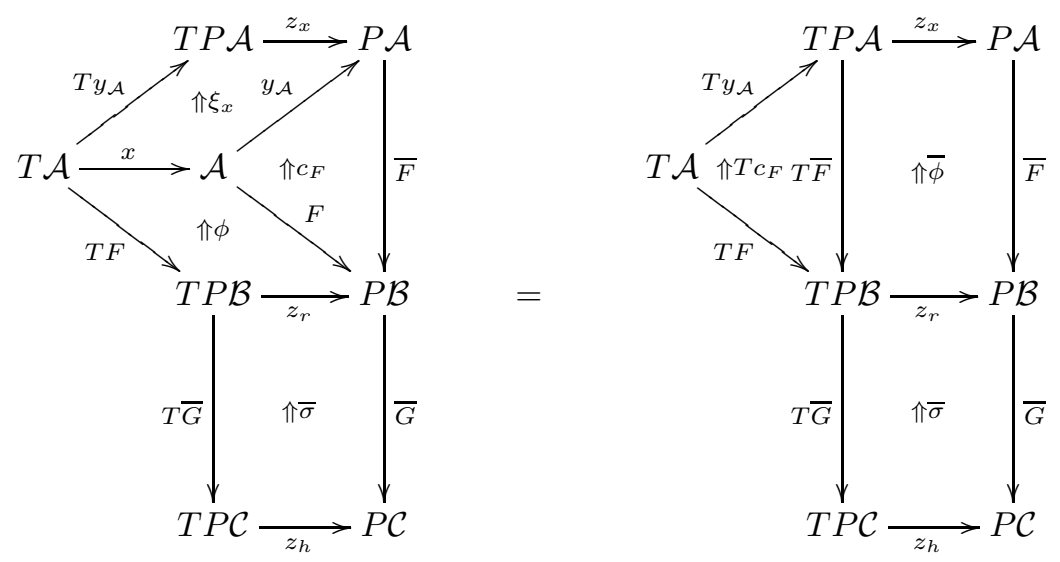

which is the defining diagram for the induced lax structure on $\bar{G} \cdot \bar{F}$ from an application of Proposition 14.

It is an easy consequence of Proposition 14 that each $\left(y_{\mathcal{A}}, \xi_{x}\right)$ is dense. Indeed since $z_{x} T$-preserves the left extension

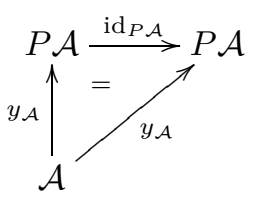

(as well the resulting left extension) the density property may be lifted to pseudo$T$-algebras applying Proposition 14.

\section{Consequences And Examples}

In this section we point out some consequences of Theorem 35 proven in the previous section, and in particular some properties of the lifted $\mathrm{KZ}$ doctrine $\widetilde{P}$ on ps- $T$-alg. Before considering the properties of $\widetilde{P}$, we mention two easy corollaries.

Corollary 49. Pseudo-distributive laws over KZ pseudomonads are essentially unique.

Proof. As shown in Lemma 41, the modification components $\omega_{2}^{\mathcal{A}}$ exhibit $\lambda_{\mathcal{A}}$ as a left extension. The last two coherence axioms of a pseudo-distributive law over a $\mathrm{KZ}$ pseudomonad then define the components $\omega_{1}^{\mathcal{A}}$ and $\omega_{3}^{\mathcal{A}}$ as unique solutions to a left extension problem. Note that $\omega_{4}^{\mathcal{A}}$ is also defined as the unique solution to a left extension problem (see the proof of 41). The essential uniqueness of left extensions then tells us these pseudo-distributive laws are essentially unique.

Corollary 50. When the conditions of Theorem 35 are met, the lifted pseudomonad arising from the pseudo-distributive law is automatically $K Z$.

Proof. As a consequence of the essential uniqueness of pseudo-distributive laws over KZ pseudomonads, any lifted pseudomonad must be equivalent to the $\mathrm{KZ}$ pseudomonad whose existence is guaranteed by Theorem 35 . 
4.1. The Lifted KZ Doctrines. We first check that in addition to having a lifting to ps- $T$-alg, we have a lifting to the 2 -category of pseudo- $T$-algebras, lax (or oplax) $T$-morphisms, and $T$-transformations.

Proposition 51. Suppose any of the equivalent conditions of Theorem 35 are satisfied. Then

(a) $P$ lifts to a $K Z$ doctrine $\widetilde{P}_{\text {oplax }}$ on $p s-T$-alg oplax $_{\text {; }}$

(b) $P$ lifts to a $K Z$ doctrine $\widetilde{P}_{\text {lax }}$ on $p s-T$-alg $g_{\text {lax }}$;

Proof. (a) : $P$ lifts to a KZ doctrine $\widetilde{P}_{\text {oplax }}$ on ps- $T$-alg $\operatorname{loplax}_{\text {since given any oplax }}$ structure cell $\varphi$ on a map $F: \mathcal{A} \rightarrow P \mathcal{B}$ as below

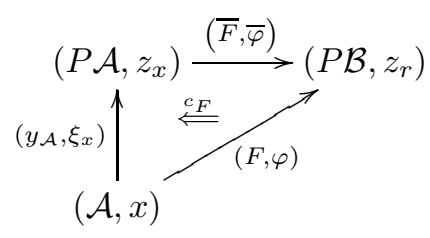

we get an oplax structure cell $\bar{\varphi}$ given as unique the solution to

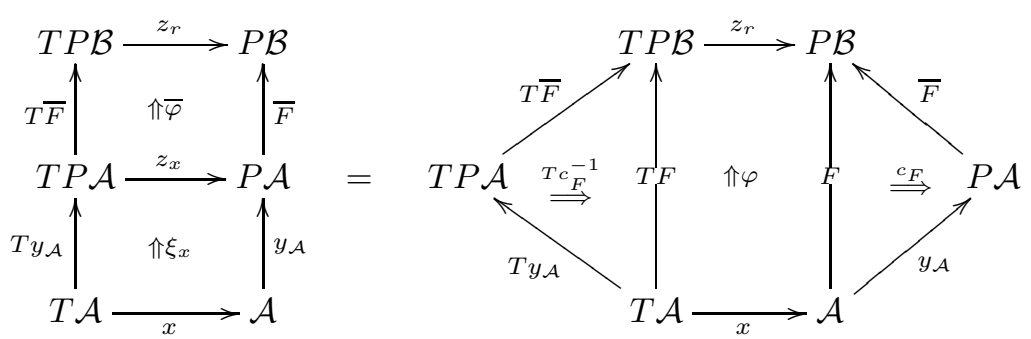

with the coherence conditions for $\bar{\varphi}$ being an oplax $T$-morphism structure following from Proposition 45 (Part 4). Note that the induced oplax structure when composed by an oplax $T$-morphism $(\bar{G}, \bar{\tau})$ as below

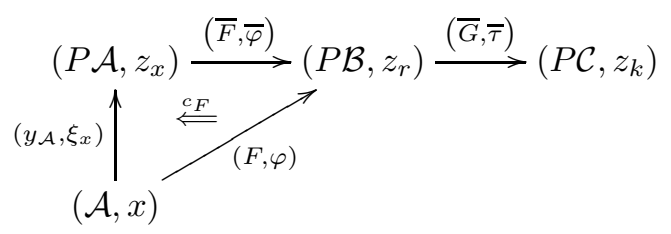

is still $(\bar{G}, \bar{\tau}) \cdot(\bar{F}, \bar{\varphi})$. To see that $(\bar{F}, \bar{\varphi})$ is a left extension in the sense of transformations, suppose we are given a transformation $\sigma:(F, \varphi) \rightarrow(H, \psi) \cdot\left(y_{\mathcal{A}}, \xi_{x}\right)$, then the induced cell $\bar{\sigma}: \bar{F} \rightarrow H$ is a transformation since

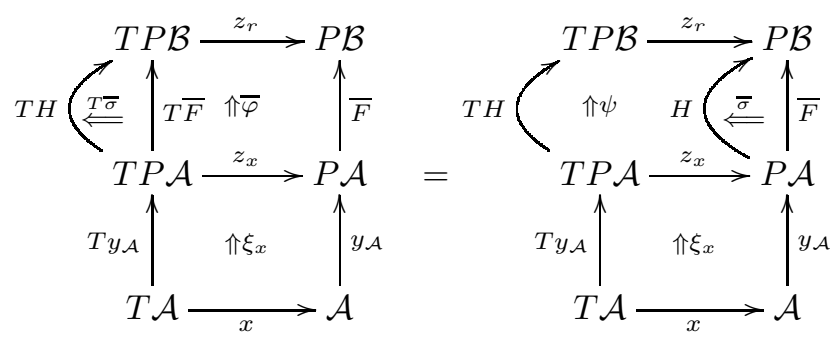

as a consequence of $\sigma$ being a transformation. By Proposition 14 the density property is still valid in the setting of oplax $T$-morphisms; this being why we proved the general case of Proposition 14 in terms of composites of lax and oplax morphisms.

(b) : The proof that $P$ lifts to a KZ doctrine $\widetilde{P}_{\text {lax }}$ on ps- $T$-alg $\lg _{\text {lax }}$ is essentially given in Theorem 48. 
We now check that the $\mathrm{KZ}$ structure cell $\theta: P y \rightarrow y P$ remains the same upon lifting to algebras.

Proposition 52. Suppose any of the equivalent conditions of Theorem 35 are satisfied. Then the KZ structure cell $\theta: P y \rightarrow y P$ for $P$ is also the $K Z$ structure cell for $\widetilde{P}$.

Proof. Recall that the components of $\theta$ are recovered as the induced cells out of the left extensions $P y_{\mathcal{A}}$ as in the diagram below

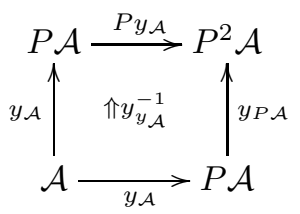

such that the composite with this diagram is an identity. Now apply Proposition 14 to this naturality square noting that each $y_{\mathcal{A}}$ extends to a pseudo $T$-morphism $\left(y_{\mathcal{A}}, \xi_{x}\right)$ in order to recover the components of the KZ structure cell for $\widetilde{P}$.

If we are to study the lifted $\mathrm{KZ}$ doctrine $\widetilde{P}$, we should consider the $\widetilde{P}$-cocomplete objects and the $\widetilde{P}$-admissible maps. We start with the former.

Algebraic cocompleteness is usually defined by asking that the underlying object be cocomplete, and that the algebra structure map be separately cocontinuous. The following proposition justifies this definition.

Proposition 53. Suppose any of the equivalent conditions of Theorem 35 are satisfied. Then a pseudo T-algebra $(\mathcal{A}, x)$ is

(a) $\widetilde{P}$-cocomplete iff $\mathcal{A}$ is $P$-cocomplete and $x: T \mathcal{A} \rightarrow \mathcal{A}$ is $T_{P}$-cocontinuous;

(b) $\widetilde{P}_{\text {lax }}$-cocomplete iff $\mathcal{A}$ is $P$-cocomplete and $x: T \mathcal{A} \rightarrow \mathcal{A}$ is $T_{P}$-cocontinuous;

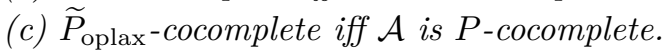

Moreover, the pseudo/lax/oplax T-morphisms $(F, \phi)$ which are $\widetilde{P} / \widetilde{P}_{\text {lax }} / \widetilde{P}_{\text {oplax }}$-cocontinuous are all classified by those maps for which the underlying $F$ is $P$-cocontinuous.

Proof. We start off by proving part $(a)$.

$(\Longrightarrow)$ : Suppose that $(\mathcal{A}, x)$ is a $\widetilde{P}$-cocomplete pseudo $T$-algebra. Then, by doctrinal adjunction [13], the pseudo $T$-morphism $\left(y_{\mathcal{A}}, \xi_{x}\right)$ has a reflection left adjoint $\left(\left(y_{\mathcal{A}}\right)_{*},\left(\xi_{x}^{-1}\right)_{*}\right)$ for which $\left(\xi_{x}^{-1}\right)_{*}$ is defined by the mates correspondence and is invertible. That is, we have isomorphisms
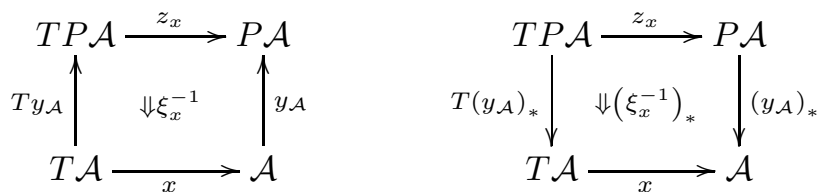

Now $\left(y_{\mathcal{A}}\right)_{*} \dashv y_{\mathcal{A}}$ via a reflection adjoint so $\mathcal{A}$ is $P$-cocomplete. We thus check that $x: T \mathcal{A} \rightarrow \mathcal{A}$ is $T_{P}$-cocontinuous. Suppose we are given a left extension as on the left
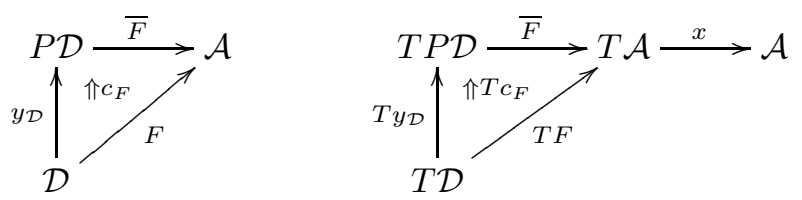
We check that the right diagram is a left extension. We first note this is equivalent to showing that $x T$-preserves left extensions as on the left below
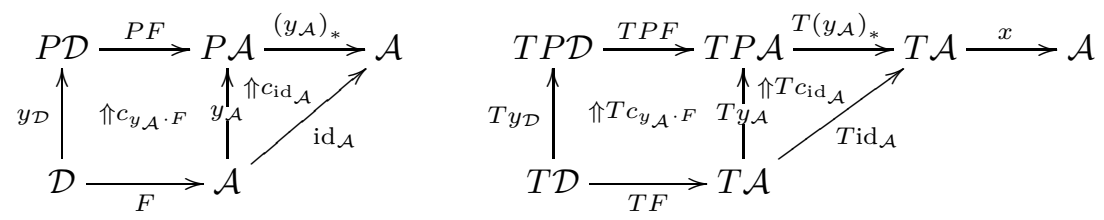

and so it suffices to check the right diagram is a left extension. This is seen upon pasting with the isomorphism $\left(\xi_{x}^{-1}\right)_{*}$ as $z_{x}$ is $T_{P}$-cocontinuous and $\left(y_{\mathcal{A}}\right)_{*}$ is a left adjoint (and hence preserves all left extensions).

$(\Longleftarrow)$ : Suppose that $\mathcal{A}$ is $P$-cocomplete and $x$ is $T_{P}$-cocontinuous. Then $(\mathcal{A}, x)$ is $\widetilde{P}$-cocomplete as $(\mathcal{A}, x)$ admits left extensions along $\left(y_{\mathcal{A}}, \xi_{x}\right)$ by Proposition 14 , and showing that such left extensions admit a pseudo $T$-morphism structure and are preserved is a similar calculation to that in the proof of Theorem 48 .

(b) : The proof of the classification of $\widetilde{P}_{\text {lax }}$-cocomplete pseudo $P$-algebras is almost the same (as the reflection left adjoint must again be pseudo by doctrinal adjunction [13]), and so we omit the details.

(c) : The $\widetilde{P}_{\text {oplax }}$-cocomplete pseudo $P$-algebras are those with an underlying $P$-cocomplete object, as a consequence of doctrinal adjunction [13].

That the $T$-morphisms $(F, \phi)$ which are $\widetilde{P} / \widetilde{P}_{\text {lax }} / \widetilde{P}_{\text {oplax }}$-cocontinuous are all classified by those morphisms for which the underlying $F$ is $P$-cocontinuous is a straightforward calculation. Indeed, given a pseudo $T$-morphism $(F, \phi)$ for which $F$ is $P$-cocontinuous, checking that $(F, \phi)$ is then $\widetilde{P}$-cocontinuous requires only checking a coherence condition (similar to 3.8$)$. Conversely, given that $(F, \phi)$ is $\widetilde{P}_{\text {- }}$ cocontinuous, that is, a pseudo $\widetilde{P}$-morphism on ps- $T$-alg, we know the underlying $F$ must be a pseudo $P$-morphism on $\mathscr{C}$ (by forgetting that certain morphisms and 2-cells are $T$-algebraic), so that $F$ is $P$-cocontinuous. The $\widetilde{P}_{\text {lax }}$ and $\widetilde{P}_{\text {oplax }}$ case may be similarly seen.

Proposition 54. Suppose any of the equivalent conditions of Theorem 35 are satisfied. Assume $(L, \alpha):(\mathcal{A}, x) \rightarrow(\mathcal{B}, y)$ is a pseudo T-morphism and $L: \mathcal{A} \rightarrow \mathcal{B}$ is $P$-admissible. Then $(L, \alpha)$ is $\widetilde{P}$-admissible if and only if for every $\widetilde{P}$-cocomplete pseudo $T$-algebra $(\mathcal{C}, z)$ and pseudo $T$-morphism $(I, \xi)$ as in the diagram

$$
\begin{aligned}
(\mathcal{B}, y) \stackrel{(R, \beta)}{\longrightarrow}(\mathcal{C}, z) \\
\stackrel{\delta}{=} \uparrow_{(I, \xi)}^{\longrightarrow}(\mathcal{A}, x)
\end{aligned}
$$

the induced lax structure cell $\beta$ on the underlying left extension $R$ as in Proposition 14 is invertible. Moreover, for pseudo, lax and oplax $(L, \alpha)$ respectively,

1. $(L, \alpha)$ is $\widetilde{P}$-admissible iff $\widetilde{P}(L, \alpha)$ has a pseudo right adjoint;

2. $(L, \alpha)$ is $\widetilde{P}_{\text {lax }}$-admissible iff $\widetilde{P}(L, \alpha)$ is pseudo;

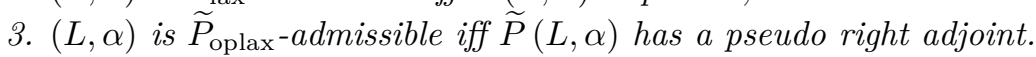

Proof. The first part of this proposition follows an equivalent characterization of $P$-admissibility as given by Bunge and Funk (discussed in [2, 29]), along with Proposition 14. The last three properties are a direct consequence of doctrinal adjunction [13]. 
Remark 55. Note that the conditions of $\widetilde{P} / \widetilde{P}_{\text {oplax-admissibility are analogous to }}$ asking a Guitart exactness condition is satisfied [9] (in the presence of some additional structure, and in the context of pointwise left extensions). However, we omit discussion of this as it would take us beyond the scope of this paper.

Remark 56. Note that if $P$ (and thus $\widetilde{P}$ ) is locally fully faithful, and $(L, \alpha)$ is a lax $T$-morphism, then $\widetilde{P}(L, \alpha)$ being pseudo implies $(L, \alpha)$ is. Indeed, the lax structure cell $\alpha$ when whiskered by $y_{\mathcal{A}}$ is invertible (a direct consequence of how the structure cell of $\widetilde{P}(L, \alpha)$ is defined in Proposition 14). As $y_{\mathcal{A}}$ is fully faithful, this means $\alpha$ is invertible. Hence, in this case, Statement 2 of the above proposition is equivalent to saying $(L, \alpha)$ is pseudo.

Given a $\mathrm{KZ}$ doctrine $P$ on a 2-category $\mathscr{C}$ we have an equivalence given by composition with the unit $y_{\mathcal{A}}$, namely $\mathscr{C}_{\text {ccts }}(P \mathcal{A}, \mathcal{B}) \simeq \mathscr{C}(\mathcal{A}, \mathcal{B})$, with $\mathscr{C}_{\text {ccts }}(P \mathcal{A}, \mathcal{B})$ containing left extensions of maps $\mathcal{A} \rightarrow \mathcal{B}$ along the unit $y_{\mathcal{A}}$. This is clearly essentially surjective as for an $F: \mathcal{A} \rightarrow \mathcal{B}$ we may take $\bar{F}: P \mathcal{A} \rightarrow \mathcal{B}$, and fully faithful as $y_{\mathcal{A}}$ is dense. We can thus recover Im and Kelly's following result.

Corollary 57 (Im-Kelly [10]). Suppose we are given a 2-category $\mathscr{C}$ equipped with a pseudomonad $(T, u, m)$ and a $K Z$ doctrine $(P, y)$. Suppose any of the equivalent conditions of Theorem 35 are met. Then for every pair of pseudo T-algebras $(\mathcal{A}, x)$ and $(\mathcal{B}, r)$ where $\mathcal{B}$ is $P$-cocomplete, composition with the unit $\left(y_{\mathcal{A}}, \xi_{x}\right)$ defines the equivalence

$$
\operatorname{Oplax}[(\mathcal{A}, x),(\mathcal{B}, r)] \simeq \operatorname{Oplax}_{\mathrm{ccts}}\left[\left(P \mathcal{A}, z_{x}\right),(\mathcal{B}, r)\right]
$$

where a morphism of pseudo T-algebras is cocontinuous when the underlying morphism is. Suppose further that $r$ is $T_{P}$-cocontinuous. Then composition with the unit $\left(y_{\mathcal{A}}, \xi_{x}\right)$ also defines the equivalences

$$
\begin{aligned}
\operatorname{Lax}[(\mathcal{A}, x),(\mathcal{B}, r)] & \simeq \operatorname{Lax}_{\mathrm{ccts}}\left[\left(P \mathcal{A}, z_{x}\right),(\mathcal{B}, r)\right] \\
\text { Pseudo }[(\mathcal{A}, x),(\mathcal{B}, r)] & \simeq \mathbf{P s e u d o}_{\mathrm{ccts}}\left[\left(P \mathcal{A}, z_{x}\right),(\mathcal{B}, r)\right]
\end{aligned}
$$

Moreover, the above three equivalences restrict to $P$-admissible underlying morphisms.

Proof. We need only check the restriction. Note that if $\bar{L}: P \mathcal{A} \rightarrow \mathcal{B}$ is $P$-admissible then so is the composite $\bar{L} \cdot y_{\mathcal{A}} \cong L$ due to closure under composition. If $L$ is $P$ admissible, then $\bar{L}$ has a right adjoint by [29, Lemma 12], and so $P \bar{L}$ also does.

4.2. The Preorder of KZ Doctrines on a 2-Category. In the following discussion of morphisms between KZ pseudomonads and doctrines we will omit most of the details, as this would take us beyond the scope of this paper. Moreover, the calculations are quite similar to those in Section 3.

It is the goal of this subsection to show that the 2-category of KZ pseudomonads on a 2 -category $\mathscr{C}$ is biequivalent to a preorder. This is a property one might expect given the "property like structure" viewpoint [15]; and the tools of admissible maps give us a method of proving this result.

Definition 58. Given $\mathrm{KZ}$ pseudomonads $(P, y, \mu)$ and $\left(P^{\prime}, y^{\prime}, \mu^{\prime}\right)$ on a 2-category $\mathscr{C}$, a morphism of $K Z$ pseudomonads $P \Longrightarrow P^{\prime}$ (corresponding to a lifting of the identity on $\mathscr{C}$ ) consists of a pseudonatural transformation $\alpha: P \rightarrow P^{\prime}$ and an invertible modification

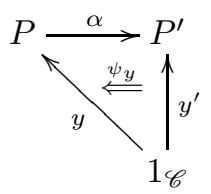


such that
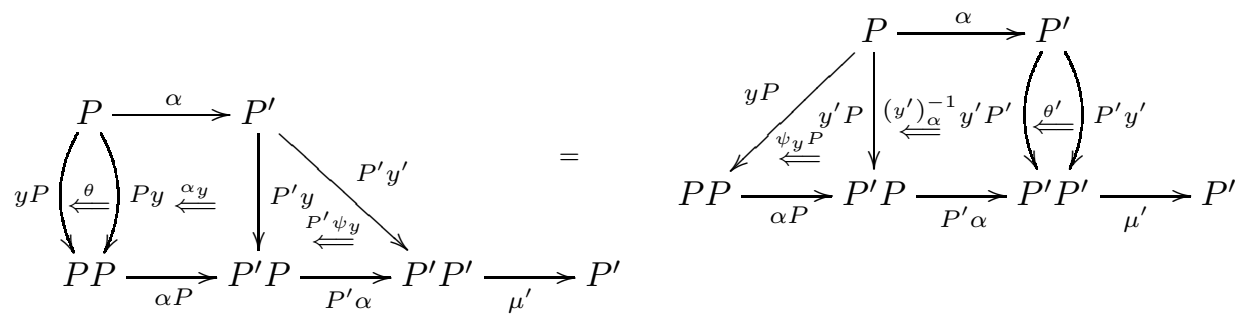

The reader will notice the following is similar to Lemma 41, meaning we are justified in omitting most of the details.

Lemma 59. Given a morphism of KZ pseudomonads as above, the 2-cell $\psi_{y}$ exhibits $\alpha$ as a left extension of $y^{\prime}$ along $y$.

Proof. We first observe that $P^{\prime} y \dashv \mu^{\prime} \cdot P^{\prime} \alpha$ (note that this right adjoint is $\bar{\alpha}$, similar to $\bar{\lambda}$ in Lemma 41) with unit $\eta$ given by

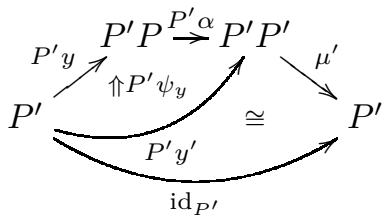

We define the counit $\varepsilon$ as the unique 2-cell for which

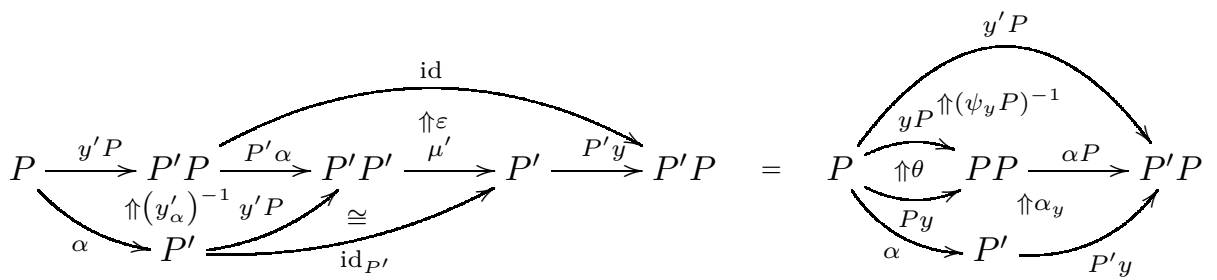

We will omit the triangle identities (as this is almost the same calculation as earlier). The result then follows from [29, Remark 16] and naturality and pseudomonad coherence axioms.

Remark 60. Given a morphism of KZ pseudomonads, we automatically have an invertible modification

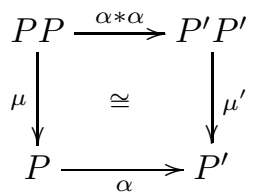

so that multiplication is respected. Indeed $\alpha \cdot \mu$ may be seen as a left extension of $y^{\prime}$ along $P y \cdot y$ exhibited by the bijections

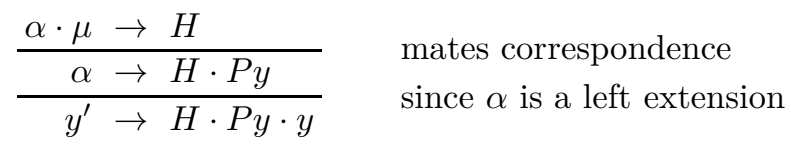

and $\mu^{\prime} \cdot \alpha * \alpha$ may be seen as left extension of $y^{\prime}$ along $y P \cdot y$ by recalling that $R_{L}=\operatorname{res}_{L} \cdot y_{\mathcal{B}}$ for admissible $L: \mathcal{A} \rightarrow \mathcal{B}$ [29, Remark 16] and taking $L$ to be an arbitrary component of $y P \cdot y$ with respect to $P^{\prime}$-admissibility. In particular, noting that $P^{\prime} y \dashv \mu^{\prime} \cdot P^{\prime} \alpha$ and $P^{\prime} y P \dashv \mu^{\prime} \cdot P^{\prime} \alpha P$ gives us the necessary data for constructing $R_{L}$. Finally, noting that $y P \cdot y \cong P y \cdot y$ gives the result. 
Definition 61. Given $\mathrm{KZ}$ doctrines $(P, y)$ and $\left(P^{\prime}, y^{\prime}\right)$ on a 2-category $\mathscr{C}$ a morphism of $K Z$ doctrines $P \Longrightarrow P^{\prime}$ consists of the assertions that:

(1) every $P$-admissible map is also $P^{\prime}$-admissible;

(2) for each $\mathcal{A} \in \mathscr{C}$, the resulting 2-cell exhibiting the left extension $\alpha_{\mathcal{A}}$

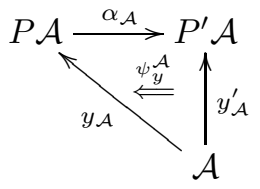

is invertible;

(3) for each $\mathcal{A}, \mathcal{B} \in \mathscr{C}$, left extensions along $y_{\mathcal{A}}$ into $P \mathcal{B}$ are preserved by $\alpha_{\mathcal{B}} .{ }^{9}$

Lemma 62. Suppose we are given two $K Z$ doctrines $(P, y)$ and $\left(P^{\prime}, y^{\prime}\right)$ on a 2category $\mathscr{C}$, with corresponding $K Z$ pseudomonads $(P, y, \mu)$ and $\left(P^{\prime}, y^{\prime}, \mu^{\prime}\right)$. Then morphisms $P \Longrightarrow P^{\prime}$ of $K Z$ doctrines are in bijection with morphisms $P \Longrightarrow P^{\prime}$ of $K Z$ pseudomonads (identified via uniqueness of left extensions up to coherent isomorphism).

Proof. Given that every $P$-admissible map is also $P^{\prime}$-admissible, we know that $P^{\prime} y$ has a right adjoint (and that we have a left extension $\alpha$ as above, assumed invertible). In particular, this right adjoint may be constructed as in [29, Prop. 15], and thus we have an adjunction $P^{\prime} y \dashv \mu^{\prime} \cdot P^{\prime} \alpha$ with unit and counit as above. The triangle identities then force the coherence condition. Pseudonaturality of $\alpha$ is equivalent to the preservation condition.

Conversely, given a morphism of KZ pseudomonads (which always gives rise to a usual morphism of pseudomonads) we know that every $P^{\prime}$-cocomplete object is also $P$-cocomplete (as the cocomplete objects may be characterized as algebras), and similarly for homomorphisms. Hence given a $P$-admissible map $L: \mathcal{A} \rightarrow \mathcal{B}$ and map $K: \mathcal{A} \rightarrow \mathcal{X}$ for a $P^{\prime}$-cocomplete (and thus also $P$-cocomplete) object $\mathcal{X}$, there exists a left extension $J: \mathcal{B} \rightarrow \mathcal{X}$ which is preserved by any $P^{\prime}$-homomorphism (as such is necessarily a $P$-homomorphism also). Consequently, $L$ must be $P^{\prime}$-admissible.

Combining this with the results of [25], yields the following proposition.

Proposition 63. Given a 2-category $\mathscr{C}$, the assignation of [25, Theorems 4.1,4.2] underlies a biequivalence

$$
\operatorname{KZdoc}(\mathscr{C}) \simeq \operatorname{KZps}(\mathscr{C})
$$

where KZps $(\mathscr{C})$ is the 2-category of $K Z$ pseudomonads, morphisms of $K Z$ pseu-

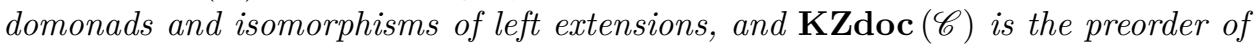
$K Z$ doctrines and morphisms of $K Z$ doctrines.

4.3. Examples. Consider the 2-monad $T$ on locally small categories for strict monoidal categories, and take $P$ to be the free small cocompletion $\mathrm{KZ}$ doctrine on locally small categories. Note that the pseudo- $T$-algebras are unbiased monoidal categories (equivalent to (strict) monoidal categories [20]) and so we may write ps- $T$-alg $\simeq$ MonCat $_{\mathrm{ps}}$ with the latter being the 2-category of monoidal categories, strong monoidal functors and monoidal transformations.

Given a monoidal category $(\mathcal{A}, \otimes)$ we may define a monoidal structure on $P \mathcal{A}$ by Day's convolution formula

$$
F \otimes_{\text {Day }} G:=\int^{a, b \in \mathcal{A}} \mathcal{A}(-, a \otimes b) \times F a \times G b
$$

\footnotetext{
${ }^{9}$ Consequently, components of $\alpha$ are $P$-homomorphisms.
} 
for small presheaves $F$ and $G$ on $\mathcal{A}$. Note that $F \otimes_{\text {Day }} G$ is then small, see [5, Section 7]. This can be shown to give a monoidal structure by the arguments of Day [4], equivalent to the structure of a pseudo- $T$-algebra. As the convolution algebra structure map is separately cocontinuous (and hence $T_{P}$-cocontinuous [32, Prop. 2.3.2]) we have enough of Proposition 45 to show condition (a) of Theorem 35 is met.

We thus know that $T$ preserves $P$-admissible maps. This says that if we suppose that $L: \mathcal{A} \rightarrow \mathcal{B}$ is $P$-admissible, meaning that each $\mathcal{B}(L-, b)$ is a small colimit of representables, then each

$$
T \mathcal{B}(T L-, \mathbf{b})=T \mathcal{B}\left[(L-, \cdots L-),\left(b_{1}, \cdots, b_{n}\right)\right]=\prod_{j=1}^{n} \mathcal{B}\left(L-, b_{j}\right)
$$

is also a small colimit of representables.

For simplicity, we will consider the preservation of the admissibility of $L=y_{\mathcal{A}}$ (which is equivalent to preservation for all $L$ ). The existence of a pseudo-distributive law of $T$ over $P$ then yields the following example.

Proposition 64. Let $X, Y: \mathcal{A}^{\mathrm{op}} \rightarrow$ Set be two small presheaves on $\mathcal{A}$. Then

$$
X \times Y:(\mathcal{A} \times \mathcal{A})^{\mathrm{op}} \rightarrow \text { Set }, \quad\left(a_{1}, a_{2}\right) \mapsto X\left(a_{1}\right) \times Y\left(a_{2}\right)
$$

is a small presheaf on $\mathcal{A} \times \mathcal{A}$.

Proof. Note that $T y_{\mathcal{A}}$ is $P$-admissible, and hence

$$
T P \mathcal{A}\left(T y_{\mathcal{A}}-, \mathbf{X}\right):(T \mathcal{A})^{\mathrm{op}} \rightarrow \text { Set }
$$

is a small presheaf on $T \mathcal{A}$ for each $\mathbf{X}=\left(X_{1}, \cdots, X_{n}\right)$ in $T P \mathcal{A}$. In particular, if we take $\mathbf{X}=(X, Y)$ then

$$
\begin{aligned}
\operatorname{TP} \mathcal{A}\left(y_{\mathcal{A}}-, \mathbf{X}\right) & = \begin{cases}\operatorname{TP} \mathcal{A}\left[\left(y_{\mathcal{A}}-, y_{\mathcal{A}}-\right),(X, Y)\right], & \mathbf{a} \in(\mathcal{A} \times \mathcal{A})^{\mathrm{op}} \\
\emptyset, & \text { otherwise }\end{cases} \\
& = \begin{cases}X(-) \times Y(-), & \mathbf{a} \in(\mathcal{A} \times \mathcal{A})^{\mathrm{op}} \\
\emptyset, & \text { otherwise }\end{cases}
\end{aligned}
$$

is a small presheaf on $\sum_{n \in \mathbb{N}} \mathcal{A}^{n}$ and so $X(-) \times Y(-)$ is a small presheaf on $\mathcal{A} \times$ $\mathcal{A}$.

Our results also apply to the less general setting of distributing (co)KZ doctrines over KZ doctrines. The following is such an example.

Example 65. Consider the KZ doctrine for the free coproduct completion

$$
\text { Fam }_{\Sigma}: \text { Cat } \rightarrow \text { Cat. }
$$

Here a map $L: \mathcal{A} \rightarrow \mathcal{B}$ is $\mathbf{F a m}_{\Sigma}$-admissible when $\mathbf{F a m}_{\Sigma} L$ is a left adjoint; that is, when $L$ is a left multiadjoint. As noted by Diers [6], this is to say that for any $Z \in \mathcal{B}$ there exists a family of morphisms $\left(h_{i}: L X_{i} \rightarrow Z\right)_{i \in \mathcal{I}}$ which is universal in the sense that given any $k: L X \rightarrow Z$ there exists a unique pair $(i, f)$ with $i \in \mathcal{I}$ and $f: X \rightarrow X_{i}$ such that $h_{i} \cdot L f=k$.

It is well known the free product completion $\mathbf{F a m}_{\Pi}$ distributes over this doctrine [25, Section 8]. Thus, as a consequence of Theorem 35, we see that if a functor $L$ is a left multiadjoint, then the functor $\operatorname{Fam}_{\Pi} L$ is a left multiadjoint also.

The following is a simple consequence of the essential uniqueness of distributive laws over KZ doctrines, shown in Corollary 49. 
Example 66. Let Prof be the bicategory of profunctors on small categories, and let PROF be the Kleisli bicategory of the free small cocompletion $\mathrm{KZ}$ doctrine $P$ on locally small categories. Clearly Prof lies inside PROF. By Corollary 49, the extension of a pseudomonad $T$ on locally small categories to the bicategory PROF is essentially unique.

\section{Liftings of Locally Fully Faithful KZ Monads}

In this section, we consider the case in which the $\mathrm{KZ}$ doctrine $P$ being lifted is locally fully faithful. The reader will recall that a $\mathrm{KZ}$ doctrine $P$ is locally fully faithful precisely when each unit map $y_{\mathcal{A}}$ is fully faithful [2].

The main goal of this section is to deduce an analogue of "Doctrinal Adjunction" on the "Yoneda structure" induced by the locally fully faithful KZ doctrine $P$. We start however with the following basic properties concerning fully faithful and $P$ fully faithful maps.

Proposition 67. Suppose any of the equivalent conditions of Theorem 35 are satisfied. Then

(a) if $y_{\mathcal{A}}$ is fully faithful for every $\mathcal{A} \in \mathscr{C}$, then every $T y_{\mathcal{A}}$ is fully faithful;

(b) $T$ preserves maps which are both $P$-admissible and $P$-fully faithful.

Proof. Firstly, note that if each $y_{\mathcal{A}}$ is fully faithful (so that $y_{T \mathcal{A}}$ is fully faithful) then so is $T y_{\mathcal{A}}$, since we have an isomorphism

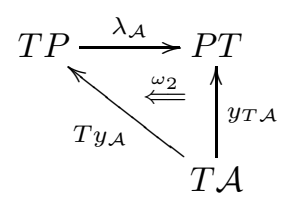

Secondly, note that if $L$ is a $P$-admissible $P$-fully faithful map, meaning the unit $\eta$ of the admissibility adjunction is invertible, then so is the unit $n$ exhibiting the admissibility of $T L$ by Figure 3.5.

5.1. Doctrinal Partial Adjunctions. In this subsection we study how pseudomonads interact with absolute left liftings (also called partial adjunctions or relative adjunctions), which we now define. In particular, we show that we get an induced oplax structure on a partial left adjoint under suitable conditions, which gives a lifting of the partial adjunction to the setting of pseudo algebras in a suitable sense.

This is in the same spirit as subsection 2.2 on algebraic left extensions, but not completely analogous (and therefore not a dual). In particular, here we do not require any algebraic cocompleteness conditions.

Definition 68. Suppose we are given a diagram of the form

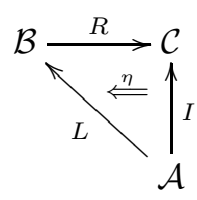

in a 2-category $\mathscr{C}$ equipped with a 2-cell $\eta: I \rightarrow R \cdot L$. We call such a diagram a partial adjunction and say that $L$ is a partial left adjoint to $R$ if given any 1-cells $M$ and $N$ as below, for any 2-cell $\zeta: I \cdot M \rightarrow R \cdot N$ there exists a unique $\bar{\zeta}: L \cdot M \rightarrow N$ 
such that $\zeta$ is equal to the pasting

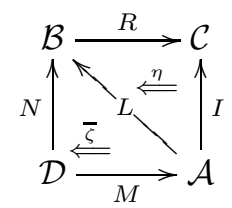

That is, pasting 2-cells of the form $\bar{\zeta}$ above with $\eta$ defines a bijection of 2-cells.

Remark 69. It is an easy and well known exercise to check that we have an adjunction $L \dashv R: \mathcal{B} \rightarrow \mathcal{A}$ with unit $\eta$ in a 2-category $\mathscr{C}$ if and only if

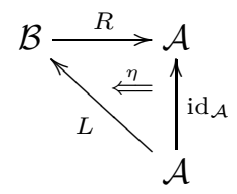

exhibits $L$ as a partial left adjoint.

We now define a notion of partial adjunction in the context of pseudo $T$-algebras and $T$-morphisms.

Definition 70. Suppose we are given oplax $T$-morphisms $(I, \xi)$ and $(L, \alpha)$ and a lax $T$-morphism $(R, \beta)$ equipped with a $T$-transformation $\eta$ (as in Remark 7 with appropriate identities) as in the diagram

$$
\begin{array}{r}
(\mathcal{B}, T \mathcal{B} \stackrel{y}{\rightarrow} \mathcal{B}) \stackrel{(R, \beta)}{\stackrel{(L, \alpha)}{\rightleftharpoons}}(\mathcal{C}, T \mathcal{C} \stackrel{z}{\rightarrow} \mathcal{C}) \\
(\mathcal{A}, T \mathcal{A} \stackrel{x}{\rightarrow} \mathcal{A})
\end{array}
$$

We call such a diagram a $T$-partial adjunction if for any given pseudo $T$-algebra $(\mathcal{D}, w)$, lax $T$-morphism $(M, \varepsilon)$, and oplax $T$-morphism $(N, \varphi)$ as below

$$
\begin{aligned}
& (\mathcal{B}, T \mathcal{B} \stackrel{y}{\rightarrow} \mathcal{B}) \stackrel{(R, \beta)}{\longrightarrow}(\mathcal{C}, T \mathcal{C} \stackrel{z}{\rightarrow} \mathcal{C}) \\
& (N, \varphi) \uparrow \stackrel{\eta}{\Longleftarrow}(L, \alpha) \stackrel{\eta}{\Longleftarrow} \uparrow_{(I, \xi)} \\
& (\mathcal{D}, T \mathcal{D} \stackrel{w}{\rightarrow} \mathcal{D}) \underset{(M, \varepsilon)}{\Longleftarrow}(\mathcal{A}, T \mathcal{A} \stackrel{x}{\rightarrow} \mathcal{A})
\end{aligned}
$$

pasting $T$-transformations of the form $\bar{\zeta}$ above with the $T$-transformation $\eta$ defines the bijection of $T$-transformations:

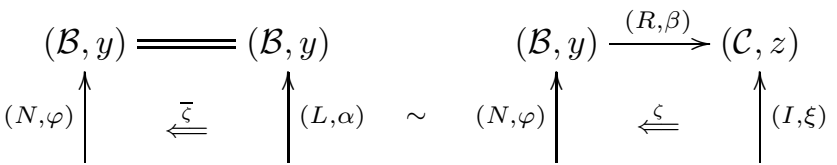

$$
\begin{aligned}
& (\mathcal{D}, w) \underset{(M, \varepsilon)}{\longrightarrow}(\mathcal{A}, x) \quad(\mathcal{D}, w) \underset{(M, \varepsilon)}{\longrightarrow}(\mathcal{A}, x)
\end{aligned}
$$

This operation of pasting the $T$-transformation $\bar{\zeta}$ with $\eta$ is given by pasting the underlying 2 -cells. The verification that such a pasting of $T$-transformations yields a $T$-transformation is a simple exercise. 
Remark 71 . We may be more general here by replacing $(M, \varepsilon)$ and $(N, \varphi)$ by a lax followed by an oplax, and an oplax followed by a lax $T$-morphism respectively. However, this level of generality will not be necessary for this paper.

We now give the doctrinal properties enjoyed by partial adjunctions.

Proposition 72. Suppose we are given a partial adjunction

$$
\mathcal{B} \stackrel{R}{\stackrel{\eta}{\rightleftharpoons}} \underset{L}{\stackrel{C}{\longrightarrow}} I
$$

in a 2-category $\mathscr{C}$ equipped with a pseudomonad $(T, u, m)$. Suppose further that

$$
(\mathcal{A}, T \mathcal{A} \stackrel{x}{\longrightarrow} \mathcal{A}), \quad(\mathcal{B}, T \mathcal{B} \stackrel{y}{\longrightarrow} \mathcal{B}), \quad(\mathcal{C}, T \mathcal{C} \stackrel{z}{\longrightarrow} \mathcal{C})
$$

are pseudo T-algebras. Then given an oplax T-morphism structure $\xi$ on $I$ and a lax $T$-morphism structure $\beta$ on $R$, there exists a unique oplax $T$-morphism structure $\alpha$ on $L$ such that $\eta$ is a T-transformation. Moreover, this partial adjunction is then lifted to the T-partial adjunction

$$
\begin{aligned}
(\mathcal{B}, T \mathcal{B} \stackrel{y}{\rightarrow} \mathcal{B}) \underset{(L, \alpha)}{\stackrel{(R, \beta)}{\longrightarrow}} & (\mathcal{C}, T \mathcal{H} \stackrel{z}{\rightarrow} \mathcal{C}) \\
& (\mathcal{A}, T \mathcal{A} \stackrel{x}{\rightarrow} \mathcal{A})
\end{aligned}
$$

Proof. Given our 2-cells
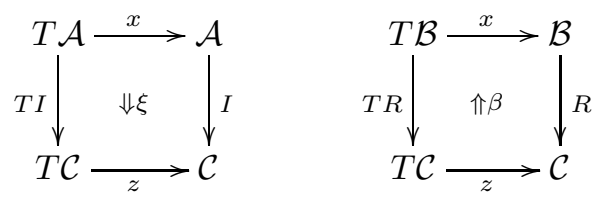

exhibiting $I$ as an oplax $T$-morphism and $R$ as a lax $T$-morphism, we can take our oplax constraint cell of $L$ (which we call $\alpha$ ) as the unique solution to
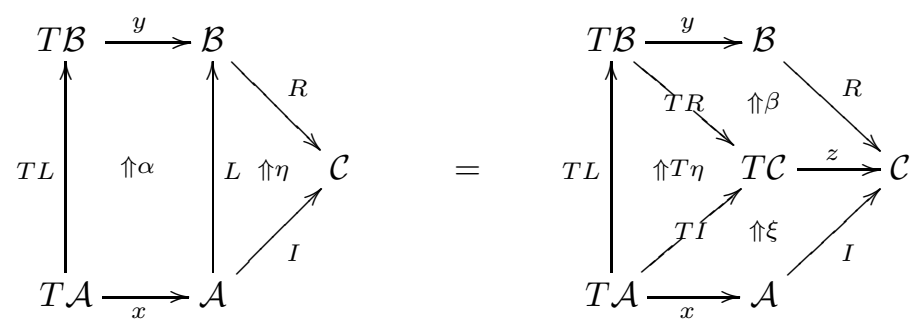

which exists since $L$ is a partial left adjoint. That is, $\alpha$ is the unique oplax structure on $L$ for which $\eta: I \rightarrow R \cdot L$ is a $T$-transformation. The verification that $\alpha$ then satisfies the unitary and multiplicative coherence axioms is a simple exercise which we omit.

The following example is an easy application of this result which does not involve Yoneda structures.

Proposition 73. Suppose $\mathscr{A}, \mathscr{B}$ and $\mathscr{C}$ are bicategories. Consider a diagram

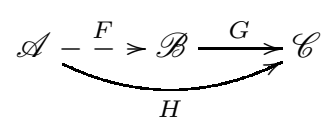


where $G$ is a lax and locally fully faithful functor, $H$ is an oplax functor, and $F$ is a locally defined functor

$$
\left(F_{X, Y}: \mathscr{A}(X, Y) \rightarrow \mathscr{B}(F X, F Y): X, Y \in \mathscr{A}\right)
$$

where $G \cdot F=H$ locally. It then follows that $F$ extends to an oplax functor.

Proof. To see this, recall that the fully faithfulness of each $G_{M, N}$ (for objects $M, N \in \mathscr{B}$ ) may be characterized by saying that each

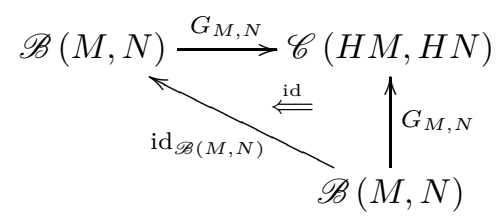

is an absolute lifting [30, Example 2.18]. As this absolute left lifting is preserved upon whiskering by

$$
F_{X, Y}: \mathscr{A}(X, Y) \rightarrow \mathscr{B}(F X, F Y)
$$

we have the family of partial adjunctions

$$
\mathscr{B}(F X, F Y) \stackrel{G_{F X, F Y}}{\longrightarrow} \mathscr{C}(H X, H Y)
$$

Endowing with the bicategory structure of $\mathscr{A}$, and full sub-bicategory structures of $\mathscr{B}$ and $\mathscr{C}$ restricted to objects in the images of $F$ and $H$ respectively, we see by Proposition 72 that $F$ extends to an oplax functor $F: \mathscr{A} \rightarrow \mathscr{B}$.

Remark 74. Clearly, this may be stated more generally in the setting of a pseudo $T$ algebras. Also, it suffices to only have an isomorphism $G F \cong H$ on the underlying 2-category.

Remark 75. In Kelly's setting of a doctrinal adjunction [13], if both the left and right adjoint are lax, exhibited by a counit and unit which are $T$-transformations of lax $T$-morphisms, then the induced oplax structure on the left adjoint is inverse to the given lax structure. In this partial adjunction case, the best we can say is that if $(I, \xi)$ is pseudo, $\left(L, \alpha^{*}\right)$ lax, and $\eta:(I, \xi) \rightarrow(R, \beta) \cdot\left(L, \alpha^{*}\right)$ a $T$-transformation of lax $T$-morphisms, then the induced oplax structure on $L$ given as $\alpha$ satisfies $\alpha^{*} \cdot \alpha=\operatorname{id}_{L \cdot x}$. This means the identity 2 -cell is a generalized $T$-transformation from $(L, \alpha)$ to $\left(L, \alpha^{*}\right)$, but not necessarily the other way around.

5.2. Doctrinal "Yoneda Structures". Kelly [13] showed that given an adjunction $L \dashv R$ which lifts to pseudo algebras, oplax structures on the left adjoint are in bijection with lax structures on the right adjoint. The goal of this section is to give a similar result for "Yoneda structure diagrams", that is diagrams of the form

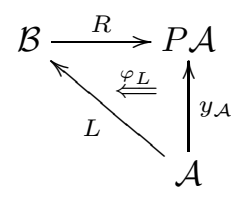

for which $L$ is an absolute left lifting, and $R$ is a left extension exhibited by the same 2-cell $\varphi_{L}$ (as appear in Yoneda structures [26], or in the setting of a locally fully faithful KZ doctrine [29]).

We state the following as one of the main results of this paper, due to its applications as a coherence result for oplax functors out of certain bicategories, such as 
the bicategories of spans or polynomials. This application will be briefly discussed at the end of this section.

Theorem 76 (Doctrinal Yoneda Structures). Suppose we are given a 2-category $\mathscr{C}$ equipped with a pseudomonad $(T, u, m)$ and a locally fully faithful $K Z$ doctrine $(P, y)$. Suppose that $T$ pseudo-distributes over $P$. Suppose we are given pseudo T-algebra structures

$$
(\mathcal{A}, T \mathcal{A} \stackrel{x}{\longrightarrow} \mathcal{A}), \quad(\mathcal{B}, T \mathcal{B} \stackrel{y}{\longrightarrow} \mathcal{B})
$$

Then for any $P$-admissible map $L: \mathcal{A} \rightarrow \mathcal{B}$ we have a Yoneda structure diagram as on the left, underlying a "doctrinal Yoneda structure" diagram as on the right
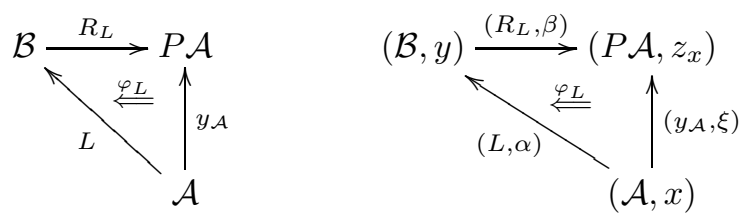

in that 2-cells $\alpha$ as on the left below exhibiting $L$ as an oplax T-morphism
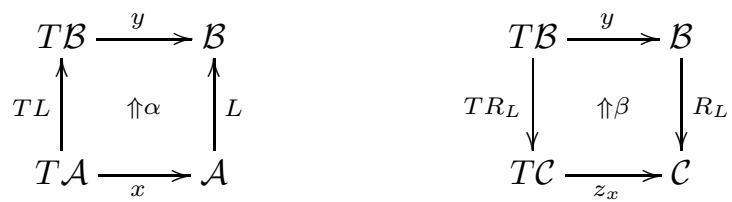

are in bijection with 2-cells $\beta$ as on the right exhibiting $R_{L}$ as a lax T-morphism.

Proof. We need only check that the propositions concerning partial adjunctions and left extensions ${ }^{10}$ are inverse to each other. But this is just a consequence of the fact that we can go between the defining equalities for these propositions
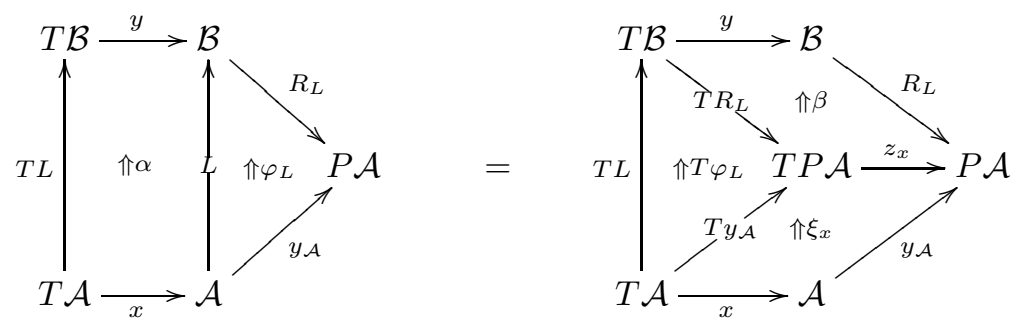

and
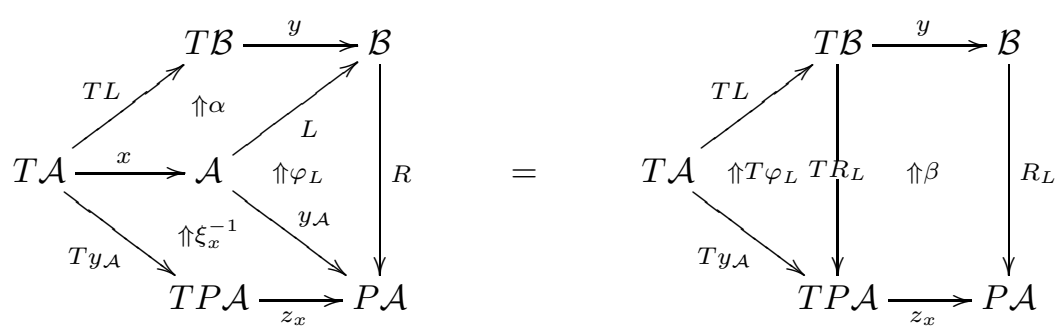

by pasting with $\xi_{x}$ and $\xi_{x}^{-1}$.

Remark 77. In the "doctrinal Yoneda structure" of the above, $\varphi_{L}$ is a $T$-transformation exhibiting $\left(R_{L}, \beta\right)$ as a $T$-left extension and $(L, \alpha)$ as a $T$-partial left adjoint, provided $\alpha$ and $\beta$ correspond via this bijection.

\footnotetext{
${ }^{10}$ Note that Proposition 14 applies since each $z_{x}$ is $T_{P}$-cocontinuous by Proposition 45.
} 
We observe that the bijection between oplax structures on left adjoints and lax structures on right adjoints as in "Doctrinal adjunction" [13] is a special case of this theorem.

Corollary 78 (Kelly). Suppose we are given a 2-category $\mathscr{C}$ equipped with a pseudomonad $(T, u, m)$, pseudo T-algebra structures

$$
(\mathcal{A}, T \mathcal{A} \stackrel{x}{\longrightarrow} \mathcal{A}), \quad(\mathcal{B}, T \mathcal{B} \stackrel{y}{\longrightarrow} \mathcal{B})
$$

and an adjunction $L \dashv R: \mathcal{B} \rightarrow \mathcal{A}$ in $\mathscr{C}$. Then oplax structures on $L$ are in bijection with lax structures on $R$.

Proof. Let $P$ be the identity pseudomonad on $\mathscr{C}$, which is clearly a locally fully faithful KZ doctrine. Trivially, any pseudomonad $T$ pseudo-distributes over the identity. Now observe that for the identity pseudomonad, the admissible maps are the left adjoints and the "Yoneda structure diagrams" are the units of adjunctions $\eta: \operatorname{id}_{\mathcal{A}} \rightarrow R \cdot L$. Applying the above theorem then gives the result.

5.3. Applications and Future Work. The motivating application of this result is not to give an analogous result to doctrinal adjunction, but instead the observation that it may be seen as a coherence result. In particular, consider the following special case of this theorem concerning the bicategory of spans in a category $\mathcal{E}$ with pullbacks, denoted $\operatorname{Span}(\mathcal{E})$.

For the following corollary, we recall that locally defined functors are the morphisms of CatGrph, and CatGrph gives rise to bicategories and oplax/lax functors via a suitable 2-monad [19].

Corollary 79. Suppose we are given a small ${ }^{11}$ category with pullbacks $\mathcal{E}$ and a bicategory $\mathscr{C}$ with the same objects, as well as locally defined functors

$$
L_{X, Y}: \operatorname{Span}(\mathcal{E})(X, Y) \rightarrow \mathscr{C}(X, Y)
$$

with corresponding left extensions $\left(R_{L}\right)_{X, Y}$ as components in the diagram

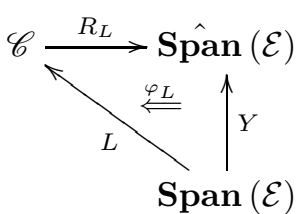

where $\hat{\operatorname{Span}}(\mathcal{E})$ is the local cocompletion ${ }^{12}$ of $\mathbf{S p a n}(\mathcal{E})$. Then oplax structures on $L$ are in bijection with lax structures on $R_{L}$.

To see why this is useful, recall that composition of spans is given by taking the terminal diagram of the form

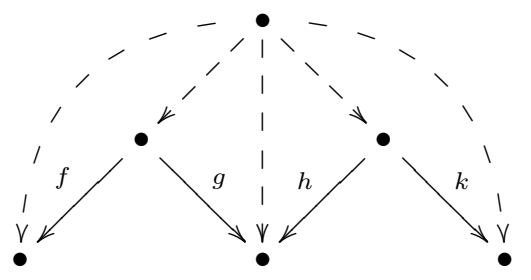

and so when evaluating the composite of two spans we may recover the two morphisms of spans in the above diagram; that is, there is a relationship between the way 2-cells are defined and how composition of 1-cells is defined.

\footnotetext{
${ }^{11}$ Note that one may work in a larger universe to work around this condition.

${ }^{12}$ The monoidal cocompletion as given by the Day convolution structure may be generalized to the setting of bicategories; we call this the local cocompletion.
} 
This relationship between composition and 2-cells is captured in Day's convolution formula [4], and causes the coend defining the Day convolution to collapse to a more workable sum. In particular, composition in $\hat{\operatorname{Span}}(\mathcal{E})$ is given by the convolution formula

$$
G F(s ; t)=\sum_{T \stackrel{h}{\longrightarrow} Y} F(s ; h) G(h ; t)
$$

where $s ; t$ is an arbitrary span from $X$ to $Z$ through $Y$, and $F$ and $G$ are presheaves on $\operatorname{Span}(\mathcal{E})(X, Y)$ and $\operatorname{Span}(\mathcal{E})(Y, Z)$ respectively. As a result, it is easier to show that a locally defined functor $L: \operatorname{Span}(\mathcal{E}) \rightarrow \mathscr{C}$ is oplax by instead showing that the corresponding $R_{L}: \mathscr{C} \rightarrow \hat{\operatorname{Span}}(\mathcal{E})$ is lax. Indeed, the reader should notice here that the problem of showing $L$ is oplax involves pullbacks, whereas the equivalent problem of showing $R$ is lax does not (once this convolution formula has been established).

A more involved application along the same lines deals not with the bicategory of spans, but instead $\mathbf{P o l y}_{c}(\mathcal{E})$, the bicategory of polynomials with cartesian 2-cells as studied by Gambino, Kock and Weber $[31,7]$. We see that due to the complicated nature of composition in $\operatorname{Poly}_{c}(\mathcal{E})$, showing that a locally defined functor $L$ : $\operatorname{Poly}(\mathcal{E}) \rightarrow \mathscr{C}$ is oplax becomes a large calculation (especially for the associativity coherence conditions); however if we instead show that $R_{L}: \mathscr{C} \rightarrow \mathbf{P o l y}_{c}(\mathcal{E})$ is lax our work will be reduced significantly; in fact by this method we can completely avoid coherences involving composition of distributivity pullbacks.

In a soon forthcoming paper we will exploit this fact in more detail to give a complete proof of the universal properties of polynomials which avoids the majority of the coherence conditions.

\section{REFERENCES}

[1] J. Beck, Distributive laws, in Sem. on Triples and Categorical Homology Theory (ETH, Zürich, 1966/67), Springer, Berlin, 1969, pp. 119-140.

[2] M. Bunge And J. Funk, On a bicomma object condition for KZ-doctrines, J. Pure Appl. Algebra, 143 (1999), pp. 69-105. Special volume on the occasion of the 60th birthday of Professor Michael Barr (Montreal, QC, 1997).

[3] E. Cheng, M. Hyland, and J. Power, Pseudo-distributive laws, Electronic Notes in Theoretical Computer Science, 83 (2003).

[4] B. DAY, On closed categories of functors, in Reports of the Midwest Category Seminar, IV, Lecture Notes in Mathematics, Vol. 137, Springer, Berlin, 1970, pp. 1-38.

[5] B. J. Day And S. Lack, Limits of small functors, J. Pure Appl. Algebra, 210 (2007), pp. 651663.

[6] Y. Diers, Catégories localisables, PhD thesis, Université de Paris VI, 1977.

[7] N. Gambino and J. Kock, Polynomial functors and polynomial monads, Math. Proc. Cambridge Philos. Soc., 154 (2013), pp. 153-192.

[8] M. Grandis and R. Pare, Adjoints for double categories, Cah. Topol. Géom. Différ. Catég., 45 (2004), pp. 193-240.

[9] R. Guitart, Relations et carrés exacts, Ann. Sci. Math. Québec, 4 (1980), pp. 103-125.

[10] G. B. Im AND G. M. Kelly, A universal property of the convolution monoidal structure, J. Pure Appl. Algebra, 43 (1986), pp. 75-88.

[11] G. M. KeLLY, On MacLane's conditions for coherence of natural associativities, commutativities, etc, J. Algebra, 1 (1964), pp. 397-402.

[12] _ Coherence theorems for lax algebras and for distributive laws, (1974), pp. 281-375. Lecture Notes in Math., Vol. 420.

[13] —, Doctrinal adjunction, in Category Seminar (Proc. Sem., Sydney, 1972/1973), Springer, Berlin, 1974, pp. 257-280. Lecture Notes in Math., Vol. 420.

[14] —, On clubs and doctrines, in Category Seminar (Proc. Sem., Sydney, 1972/1973), Springer, Berlin, 1974, pp. 181-256. Lecture Notes in Math., Vol. 420.

[15] G. M. Kelly and S. LaCK, On property-like structures, Theory Appl. Categ., 3 (1997), pp. No. 9, 213-250.

[16] A. Kock, Monads for which structures are adjoint to units, Aarhus Preprint Series, No. 35 (1972/73). Revised version published in J. Pure Appl. Algebra, 104 (1995). 
[17] S. R. Koudenburg, Algebraic Kan extensions in double categories, Theory Appl. Categ., 30 (2015), pp. 86-146.

[18] S. LACK, A 2-categories companion, in Towards higher categories, vol. 152 of IMA Vol. Math. Appl., Springer, New York, 2010, pp. 105-191.

[19] - Icons, Appl. Categ. Structures, 18 (2010), pp. 289-307.

[20] T. Leinster, Higher operads, higher categories, vol. 298 of London Mathematical Society Lecture Note Series, Cambridge University Press, Cambridge, 2004.

[21] F. Marmolejo, Doctrines whose structure forms a fully faithful adjoint string, Theory Appl. Categ., 3 (1997), pp. No. 2, 24-44.

[22] — Distributive laws for pseudomonads, Theory Appl. Categ., 5 (1999), pp. No. 5, 91-147.

[23] F. Marmolejo, R. D. Rosebrugh, and R. J. Wood, A basic distributive law, J. Pure Appl. Algebra, 168 (2002), pp. 209-226. Category theory 1999 (Coimbra).

[24] F. Marmolejo and R. J. Wood, Coherence for pseudodistributive laws revisited, Theory Appl. Categ., 20 (2008), pp. No. 5, 74-84.

[25] - Kan extensions and lax idempotent pseudomonads, Theory Appl. Categ., 26 (2012), pp. No. 1, 1-29.

[26] R. Street And R. WAlters, Yoneda structures on 2-categories, J. Algebra, 50 (1978), pp. 350-379.

[27] M. TANAKa, Pseudo-Distributive Laws and a Unified Framework for Variable Binding, PhD thesis, University of Edinburgh, 2004.

[28] W. Tholen, Lax distributive laws for topology, I. arXiv:1603.06251, 2016.

[29] C. Walker, Yoneda structures and KZ doctrines, J. Pure Appl. Algebra, 222 (2018), pp. 1375-1387.

[30] M. WeBer, Yoneda structures from 2-toposes, Appl. Categ. Structures, 15 (2007), pp. 259323.

[31] - Polynomials in categories with pullbacks, Theory Appl. Categ., 30 (2015), pp. No. 16, 533-598.

[32] — Algebraic Kan extensions along morphisms of internal algebra classifiers, Tbilisi Math. J., 9 (2016), pp. 65-142.

[33] V. Zöberlein, Doctrines on 2-categories, Math. Z., 148 (1976), pp. 267-279.

Department of Mathematics, Macquarie University, NSW 2109, Australia

E-mail address: charles.walker1@mq.edu.au 